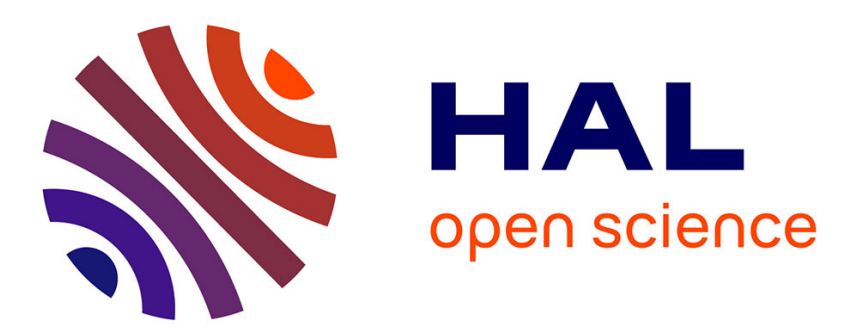

\title{
A stochastic formulation for the drag force based on multiscale numerical simulation of fluidized beds
}

Amir Esteghamatian, Florian Euzenat, Abdelkader Hammouti, Michel Lance, Anthony Wachs

\section{- To cite this version:}

Amir Esteghamatian, Florian Euzenat, Abdelkader Hammouti, Michel Lance, Anthony Wachs. A stochastic formulation for the drag force based on multiscale numerical simulation of fluidized beds. International Journal of Multiphase Flow, 2018, 99, pp.363 - 382. 10.1016/j.ijmultiphaseflow.2017.11.003 . hal-01905239

\section{HAL Id: hal-01905239 \\ https: / hal-ifp.archives-ouvertes.fr/hal-01905239}

Submitted on 25 Oct 2018

HAL is a multi-disciplinary open access archive for the deposit and dissemination of scientific research documents, whether they are published or not. The documents may come from teaching and research institutions in France or abroad, or from public or private research centers.
L'archive ouverte pluridisciplinaire HAL, est destinée au dépôt et à la diffusion de documents scientifiques de niveau recherche, publiés ou non, émanant des établissements d'enseignement et de recherche français ou étrangers, des laboratoires publics ou privés. 


\title{
A stochastic formulation for the drag force based on multiscale numerical simulation of fluidized beds
}

\author{
Amir Esteghamatian ${ }^{b, c}$, Florian Euzenat ${ }^{a}$, \\ Abdelkader Hammouti ${ }^{a}$, Michel Lance ${ }^{\mathrm{d}}$, Anthony Wachs b,c,* \\ a IFP Energies nouvelles, Fluid Mechanics Department, Rond-point de l'Echangeur \\ de Solaize, BP 3, 69360 Solaize, France \\ ${ }^{\mathrm{b}}$ Department of Mathematics, University of British Columbia, 1984 Mathematics \\ Road, Vancouver, BC, Canada V6T $1 Z 4$ \\ ${ }^{\mathrm{c}}$ Department of Chemical $\&$ Biological Engineering, University of British \\ Columbia, 2360 East Mall, Vancouver, BC, Canada V6T $1 Z 3$ \\ ${ }^{\mathrm{d}}$ Laboratoire de Mécanique des Fluides et d'Acoustique (LMFA), Ecole Centrale de \\ Lyon,36 avenue Guy de Collongue, 69134 Ecully, France
}

\begin{abstract}
In Esteghamatian, A., Bernard, M., Lance, M., Hammouti, A. and Wachs, A., 2017, Micro/meso simulation of a fluidized bed in a homogeneous bubbling regime, International Journal of Multiphase Flow, 92, 93-111, we performed a one-to-one comparison of micro-scale Particle-Resolved Simulation (PRS) results and meso-scale two-way coupled Euler-Lagrange (a. k. a. DEM-CFD for Discrete Element MethodComputational Fluid Dynamics) simulation results in a homogeneous bi-periodic liquid/solid fluidized bed. These data showed an acceptable agreement between micro- and meso-scale predictions for integral measures as, e.g., pressure drop and bed height. However, particles fluctuations are markedly underpredicted in DEMCFD simulations, especially in the direction transverse to the main flow. The filtered DEM-CFD porosity appears to be a coarse descriptor of the local microstructure and
\end{abstract}


hence is the primary reason for the inability of the meso-scale DEM-CFD model to fully recover the actual features of the flow. In this paper, we explore two different directions in improving the meso-scale model: (a) we adopt an improved inter-phase coupling scheme and (b) we introduce a stochastic formulation for the drag law derived from our PRS results. The new stochastic drag law, which incorporates information on the first and second-order moments of PRS results, shows promises to recover the appropriate level of particles fluctuations.

Key words: Multi-scale simulation; Fluidized Bed; Stochastic drag law; Discrete Element method; Fictitious Domain method; Euler/Lagrange method

\section{Introduction}

Owing to their wide presence in environmental phenomena, pharmaceutical industry, energy sector, chemical processes, biological flows, etc., particle-laden flows have been subject of extensive analytical, numerical and experimental studies. Thanks to substantial advances in the computing resources, numerical approaches attracted much attention in the past decades. Accordingly, more care is given to the development of more advanced numerical models with higher levels of accuracy.

The nonlinear and multi-scale nature of fluid/particle interactions in such systems highlights the rich dynamics of the system and calls for well-adapted numerical models. A variety of numerical models depending on the required computational resources and desired level of accuracy have been proposed in the literature (Van der Hoef et al. [2008], Tenneti and Subramaniam [2014]).

\footnotetext{
* Corresponding author.

Email address: wachs@math.ubc.ca (Anthony Wachs).
} 
At the most refined end of the spectrum, micro-scale models based on PRS are able to resolve the flow at the scale of the particle boundary layer. Meso-scale DEM-CFD models filter the fluid field at scales at least one order of magnitude larger than the particle volume. Closure laws are hence required to estimate the momentum transfer between particle phase and fluid phase. Traditionally, this filtering operation is implicit as the volume of fluid grid cells is usually much larger than the particle volume. As contributions from a Lagrangian particle are simply distributed to the Eulerian fluid grid cells intersected by this particle, the filter size implicitly spans a maximum of two fluid grid cells in each direction. Over the past few years, a new and enhanced approach that decouples the filtering kernel from fluid grid cells has been developed (Link et al. [2005], Pepiot and Desjardins [2012], Capecelatro and Desjardins [2013]). This methodology provides the model with the flexibility of using fluid grid cells with volumes comparable to the particle volume, or even smaller. The filter size is typically a few particle diameters and spans the required number of fluid grid cells as controlled by the particle diameter to fluid grid size ratio. At the coarsest end of the spectrum, macro-scale models (either Two-Fluid models or moments based methods, see Fox [2012] for more details) consider both particle phase and fluid phase as continuous media described by Eulerian transport equations. In the particular framework of Two-Fluid models, the kinetic theory of granular media is employed to consider particle-particle and particle-wall interactions.

Meso-scale models have gained a large popularity in simulation of dense fluidparticle systems during the past two decades (Tsuji et al. [1993], Pepiot and Desjardins [2012]). In contrast to Two-Fluid models, a major source of inaccuracy is eliminated by directly taking into account particle-particle and particle-wall interactions. Also, employing closure laws for fluid-particle mo- 
mentum transfer leads to a drastic reduction of the computational load (by several orders of magnitude) as compared to micro-scale models. The reasonable compromise between level of accuracy and required computational effort favors this type of models in the simulation of meso-scale systems, i.e., systems that comprise $10^{3}$ to $10^{9}$ particles and the upper limit is increasing with advances in parallel algorithms, High Performance Computing and computational resources.

While meso-scale models have been widely applied to fluid-particle simulations since the 90s, only recently attention has been given to the accuracy of the model, particularly in terms of predictions of second-order particle statistics (Subramaniam et al. [2014], Kriebitzsch et al. [2013], Esteghamatian et al. [2017a]). Since the fluid field remains unresolved at the level of particles, particle-induced fluctuations and in turn particle agitations are suppressed to some extent by meso-scale models. Subramaniam et al. [2014] emphasized the underprediction of particles acceleration by point-particle models in decaying isotropic turbulent flow. Kriebitzsch et al. [2013] also pointed out the underestimation of the drag force in DEM-CFD simulations as compared to PRS. In our previous work (Esteghamatian et al. [2017a]), we also observed the underestimation of the particles granular temperature particularly in the direction transverse to the mean flow.

In Esteghamatian et al. [2017b], we further analyzed the PRS of fluidization to have a better insight into the dominant mechanisms of particles motion. In short, we have so far drawn the following conclusions: (i) the meso-scale model partially resolves fluid fluctuations and underpredicts particles fluctuations, and (ii) local fluid fluctuations around the particles depend on the filter size and smoothly increase with the averaging control volume particularly in a 
homogeneous system. (i) and (ii) emphasize that any information transfer from PRS to the meso-scale model requires to take into account the effect of the filter size. Strictly speaking, fluctuations captured by the meso-scale model are limited by the size of the filter. In that sense, the meso-scale model can be considered as the equivalent of the Large Eddy Simulation (LES) approach in an analogy with single-phase turbulence modeling. However, contrary to singlephase turbulence where instabilities are generated at the resolved scales, smallscale instabilities - also called pseudo-turbulence - are generated at the particle scale which is inherently not accessible with meso-scale model. The lack of a self-similar pattern and the generation of instabilities at unresolved scales prevent us from developing a universal formalism that relates momentum transfer to mean values at the filter scale. This has been previously addressed in the literature and accordingly, closures based on PRS or experiments have been proposed to correlate the mean momentum transfer to integral measures (Hill et al. [2001], Ergun [1952], Di Felice [1994]). We hope that a similar procedure can be employed to recover momentum transfer fluctuations. Given that the PRS solution already accounts for all the relevant spatial scales of the system, it is technically possible to feed the meso-scale model with the missing information at the particle level in a multi-scale framework.

The scope of the present study is to answer the following questions: (a) to which extent the performance of meso-scale models are limited by the fluid field discretization?, (b) is there any methodology to quantify the sub-grid scale fluctuations from PRS results ? To answer (a), we perform a grid refinement study with our meso-scale model. A basic feature of many classical meso-scale models (including ours in Bernard et al. [2016] Esteghamatian et al. [2017a]) is that the fluid grid cell is used as the fluid/particle averaging kernel. While pretty efficient when particles are much smaller than the fluid grid 
cell, it causes numerical stability issues when the size of particles are larger than (or even comparable to) the fluid grid cell. As suggested by Capecelatro and Desjardins [2013], it is technically possible to decouple the fluid/particle averaging kernel from the fluid grid cell. We have adopted this approach to attain the flexibility of using fine grid cells and perform a full grid refinement study. In an effort to answer (b), we have characterized the drag coefficient experienced by particles in PRS as a Probability Density Function, in contrast to the classical deterministic drag laws. This provides us with a basis for our stochastic formulation of the drag force. Stochastic approaches have been widely applied in turbulence modeling particularly involving reactive flows (Dreeben and Pope [1998] Pope [1994] Muradoglu et al. [1999]). Sommerfeld and Zivkovic [1992] and Oesterle and Petitjean [1993] introduced a probabilistic approach to model inter-particle collisions inspired by the kinetic theory of gases. In spray modeling, Subramaniam [2000] proposed a statistical representation of a spray based on a stochastic point process model. Employing a stochastic approach to model the drag force is quite scarce in the literature, yet not nonexistent. In homogeneous isotropic turbulence and considering a oneway point-particle assumption, Fede et al. [2006] proposed a stochastic model to reconstruct the sub-filter fluid fluctuations based on a Langevin equation. DNS data were used to determine the constants of the model. The authors have shown that the correct level of particles kinetic energy can be attained by the LES of the fluid field and a discrete particle method incorporating a stochastic closure. A similar approach was adopted by Berrouk et al. [2007] for the LES of inertial particles in a turbulent shear flow. Andrews et al. [2005] employed an ad hoc approach to formulate a stochastic drag coefficient in DEM-CFD coarse-grid simulations of vertical risers. The authors used DEMCFD simulations with relatively refined grid sizes as a reference to determine 
the model's constants. In a more general approach, Tenneti et al. [2016] have recently proposed a stochastic formulation for the drag force based on PRS and the kinetic theory of granular flows.

The rest of the paper is organized as follows: In Section 2, we present the governing equations and numerical aspects of our numerical tool. In Section 3, we introduce the simulation parameters. Our stochastic formulation for the drag coefficient is detailed in Section 4. In Section 5, we present the main body of results in micro/meso simulations of low density ratio fluidization. A single test case of high density ratio fluidization is subsequently studied. Finally, the conclusions are summarized in Section 6.

\section{Governing equations}

Navier-Stokes and Newton-Euler equations are solved in a coupled fashion in both micro- and meso-scale models. The incompressible Newtonian fluid assumption is considered. The micro-scale model is based on the combined mass and momentum conservation equations extended in the solid phase (Glowinski et al. [2001]). The meso-scale model is based on locally averaged mass and momentum conservation equations proposed by Anderson and Jackson [1967]. In both models the solid phase is treated in a direct fashion by solving the Newton-Euler equations for each individual particle. All parameters in this paper are dimensionless except if distinguished by a "star" symbol. The following scales are employed to non-dimensionalize the set of equations: $L_{c}^{*}$ for length, $V_{c}^{*}$ for velocity, $L_{c}^{*} / V_{c}^{*}$ for time, $\rho_{f}^{*} V_{c}^{* 2}$ for pressure, $V_{c}^{* 2} / L_{c}^{*}$ for gravity acceleration, $\rho_{f}^{*} V_{c}^{* 2} / L_{c}^{*}$ for distributed Lagrange multiplier (for the micro-scale model only) and $\rho_{f}^{*} V_{c}^{* 2} L_{c}^{* 2}$ for forces. 


\subsection{Micro-scale model}

The dimensionless and non-variational form of the combined continuity and momentum equations (written for simplicity for a system with a single particle) are as follows:

$$
\begin{aligned}
& \frac{\partial \boldsymbol{u}}{\partial t}+\boldsymbol{u} \cdot \nabla \boldsymbol{u}=-\nabla p+\frac{1}{\mathcal{R} e} \nabla^{2} \boldsymbol{u}-\boldsymbol{\lambda}, \text { over } \Omega \\
& \left(\rho_{r}-1\right) V_{p} \frac{d \boldsymbol{U}}{d t}-\int_{\mathcal{P}} \boldsymbol{\lambda} d x=\sum_{j} \boldsymbol{F}_{c j}+\mathcal{F} r\left(\rho_{r}-1\right) V_{p} \frac{\boldsymbol{g}^{*}}{\left|\boldsymbol{g}^{*}\right|}, \text { over } \mathcal{P} \\
& \left(\rho_{r}-1\right)\left(\boldsymbol{I}_{p} \frac{d \boldsymbol{\omega}}{d t}+\boldsymbol{\omega} \times \boldsymbol{I}_{p} \cdot \boldsymbol{\omega}\right)-\int_{\mathcal{P}} \boldsymbol{r} \times \boldsymbol{\lambda} d x=\sum_{j} \boldsymbol{R}_{j} \times \boldsymbol{F}_{c j}, \text { over } \mathcal{P} \\
& \boldsymbol{u}-(\boldsymbol{U}+\boldsymbol{\omega} \times \boldsymbol{r})=0, \text { over } \mathcal{P} \\
& \nabla \cdot \boldsymbol{u}=0, \text { over } \Omega
\end{aligned}
$$

where $\boldsymbol{u}, \boldsymbol{U}, \boldsymbol{\omega}$ stand for the fluid velocity vector, the particle translational velocity vector and the particle angular velocity vector, respectively. The particle domain and whole fluid/particle domain are denoted by $\mathcal{P}$ and $\Omega$, respectively. $\boldsymbol{\lambda}$ represents the distributed Lagrange multiplier vector, $\boldsymbol{F}_{c}$ the contact force, $V_{p}=M_{p}^{*} /\left(\rho_{s}^{*} L_{c}^{* d}\right)$ the particle volume, $M_{p}^{*}$ the particle mass, $d$ the dimension

of the system, $\boldsymbol{I}_{\boldsymbol{p}}=\boldsymbol{I}_{\boldsymbol{p}}^{*} / \rho_{s}^{*} L_{c}^{* d+2}$ the particle inertia tensor, $\boldsymbol{r}$ the position vector and $\boldsymbol{R}$ the vector connecting the gravity center and the contact point. The 
following dimensionless numbers are also introduced:

$$
\begin{aligned}
& \text { Reynolds number } \mathcal{R} e=\frac{\rho_{f}^{*} V_{c}^{*} L_{c}^{*}}{\mu^{*}} \\
& \text { Inverse Froude number } \mathcal{F} r=\frac{g^{*} L_{c}^{*}}{V_{c}^{* 2}} \\
& \text { Density ratio } \rho_{r}=\frac{\rho_{s}^{*}}{\rho_{f}^{*}} \\
& \text { Stokes number } \mathcal{S} t k=\frac{\rho_{r} \mathcal{R} e_{p}}{18}
\end{aligned}
$$

Where $\mu^{*}$ denotes the fluid viscosity. Please note that the Stokes number $\mathcal{S} t k$ is not an independent parameter and is given above for the sake of completeness only.

\subsection{Meso-scale model}

The Navier-stokes equations are locally averaged based on the set of equations proposed by Anderson and Jackson [1967]. Depending on the size of particles with respect to the fluid grid cells, the averaging process can be performed on either the fluid grid cells or an averaging kernel whose length scale is larger than the fluid grid cells. In both cases, the volume scale of the averaging operation is at least one order of magnitude larger than the particles diameter. The dimensionless fluid conservation equations read:

$$
\begin{aligned}
& \frac{\partial \varepsilon}{\partial t}+\nabla \cdot \varepsilon \boldsymbol{u}=0 \\
& \frac{\partial \varepsilon \boldsymbol{u}}{\partial t}+\nabla \cdot(\varepsilon \boldsymbol{u} \boldsymbol{u})=-\nabla p-\boldsymbol{F}_{f p}+\frac{1}{\mathcal{R} e} \nabla \cdot(2 \varepsilon \boldsymbol{D})
\end{aligned}
$$

where $\boldsymbol{D}$ and $\varepsilon$ denote the strain rate tensor and the fluid volume fraction, respectively. The pressure gradient term contains the hydrodynamic pressure only and $\boldsymbol{F}_{f p}$ represents the fluid/particle momentum transfer. Please note 
that in (11) we have neglected the additional unclosed Reynolds stress $\boldsymbol{R}_{\boldsymbol{u}}$ and viscous stress $\boldsymbol{R}_{\mu}$ terms that arise from volume filtering (see Capecelatro and Desjardins [2013] for more details). Since the fluid field is unresolved at the particle scales, $\boldsymbol{F}_{f p}$ needs to be closed by empirical correlations. Based on the equation proposed by Maxey and Riley [1983] and Gatignol [1983], different contributions to the momentum transfer are:

$$
\boldsymbol{F}_{f p}=\boldsymbol{F}_{d r a g}+\boldsymbol{F}_{\text {lift }}+\boldsymbol{F}_{a m}+\boldsymbol{F}_{b a}
$$

Where the right hand side terms represent drag, lift, added mass and Basset forces, respectively (Crowe et al. [1997]). The importance of each term depends on the flow configuration. For example, it can be shown that for high density ratios $\boldsymbol{F}_{a m}$ can be neglected (Crowe et al. [1997]). In Esteghamatian et al. [2017a], we have shown based on PRS data that lift and added mass contributions are negligible as compared to the drag force in a liquid/solid configuration similar to the one investigated in this work. A description of the two closure laws used in this work is presented in APPX. A.

\subsection{Newton-Euler equations and collision force}

The Newton-Euler equations solved for each individual particle read:

$$
\begin{aligned}
& \rho_{r} V_{p} \frac{d \boldsymbol{U}}{d t}=\boldsymbol{F}_{b}+\boldsymbol{F}_{c}+\boldsymbol{F}_{f p} \\
& \rho_{r}\left(\boldsymbol{I}_{p} \frac{d \boldsymbol{\omega}}{d t}+\boldsymbol{\omega} \times \boldsymbol{I}_{p} \boldsymbol{\omega}\right)=\boldsymbol{T}_{c}+\boldsymbol{T}_{f p}
\end{aligned}
$$

Where $\boldsymbol{F}_{b}=\left(\rho_{r}-1\right) \mathcal{F} r V_{p} \frac{\boldsymbol{g}}{\left|\boldsymbol{g}^{*}\right|}$ denotes the buoyancy force, $\boldsymbol{F}_{c}$ inter-particle or particle-wall contact force and $\boldsymbol{F}_{f p}$ the fluid/particle interaction force. Likewise, $\boldsymbol{T}_{c}$ and $\boldsymbol{T}_{f p}$ represent the contact-induced and the fluid-induced torque acting on the particle. A soft-sphere model is employed for the treatment of 
the inter-particle and particle-wall interactions. The model and its parallel implementation is introduced in detail in Wachs et al. [2012], Rakotonirina and Wachs [2017], Rakotonirina et al. [2017]. In short, the soft-sphere model comprises a normal elastic restoring force, a normal dissipative force and a tangential friction force. The fluid/particle interaction force $\boldsymbol{F}_{f p}$ and torque $\boldsymbol{T}_{f p}$ are computed a posteriori using the Lagrange multipliers:

$$
\begin{aligned}
\boldsymbol{F}_{f p} & =\int_{\mathcal{P}} \boldsymbol{\lambda} d \boldsymbol{x}+V_{p} \frac{d \boldsymbol{U}}{d t} \\
\boldsymbol{T}_{f p} & =\int_{\mathcal{P}}(\boldsymbol{r} \times \boldsymbol{\lambda}) d \boldsymbol{x}+\boldsymbol{I}_{p} \frac{d \boldsymbol{\omega}}{d t}+\boldsymbol{\omega} \times \boldsymbol{I}_{p} \boldsymbol{\omega}
\end{aligned}
$$

As described in equation (12), the meso-scale model employs closure laws to estimate different contributions of fluid/particle interaction forces.

\subsection{Numerical aspects}

Temporal and spatial discretization of Navier-Stokes equations in micro- and meso-scale models are described in Wachs et al. [2015], Esteghamatian et al. [2017a]. In general, for both micro- and meso-scale models the set of equations

are discretized with a second-order Finite Volume/Staggered Grid scheme. Both models employ a first order Marchuk-Yanenko operator-splitting algorithm for temporal discretization. Depending on the model, sub-problems are resolved in the following order:

(a) Micro-scale model:

(I) A classical L2-projection scheme is employed to solve the NavierStokes equations. An intermediate fluid velocity and pressure field is obtained.

(II) As a predictor step, a purely granular problem is solved and an 
intermediate particle velocity is obtained.

(III) A fictitious domain problem is solved. By the end of this subproblem, rigid-body motion is imposed in the particle domain.

(IV) As a corrector step, a purely granular problem is solved and the final particle velocity and position are computed.

(b) Meso-scale model:

(I) A modified L2-projection scheme is employed to solve Navier-Stokes equations for a known particle reaction force and a known porosity field.

(II) A granular problem with a known hydrodynamic force is solved and the updated porosity field is computed.

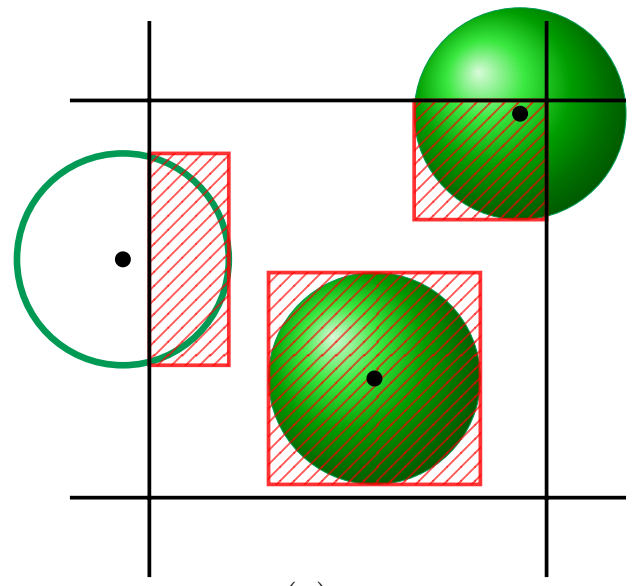

(a)

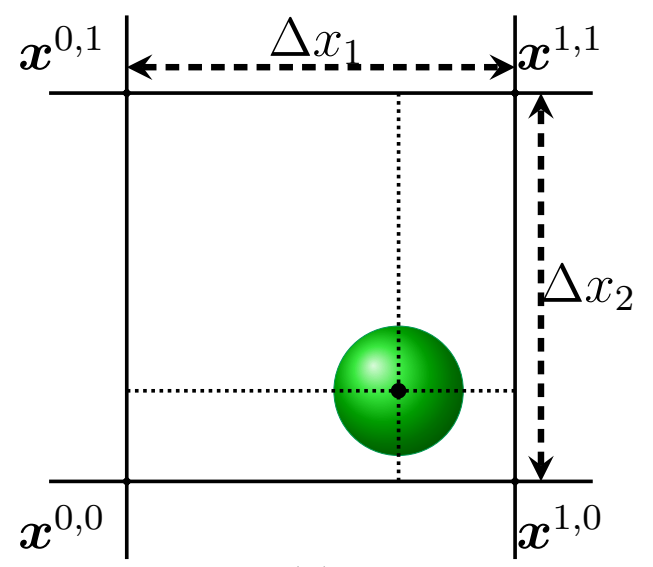

(b)

Figure 1. Bounding Cube averaging method in meso-scale model: (a) Lagrange $\rightarrow$ Euler and (b) Euler $\rightarrow$ Lagrange information transfer.

In the meso-scale model, the averaging kernel plays an important role in both the stability and the accuracy of the computed solution. In our meso-scale model we employ two different methods to compute locally averaged quantities such as porosity and slip velocity. The choice of the method depends on the ratio between the grid cell size $\Delta x$ and particles diameter $d$ :

(a) Bounding Cube (BC) method: If $\Delta x \geq 1.7 d$, we use the fluid grid cell for Euler $\rightleftarrows$ Lagrange information transfer. A bounding cube method, as 
described in Bernard et al. [2016] and schematically illustrated in FiG. 1 is used to compute the porosity field and distribute the particle reaction forces at the fluid grid cells. Additionally, a simple linear interpolation is used to transfer the information from the fluid grid cells to the particles centers.

(b) Gaussian Kernel (GK) method: If $\Delta x<1.7 d$, a truncated Gaussian kernel is used to transfer information from the fluid grid cells to the particles centers, back and forth. The main motivation behind employing this method is to decouple the fluid discretization from the averaging kernel. Furthermore, it smooths out the presence of particles on the background grid in a monotone fashion. This method is totally equivalent to the one proposed in Capecelatro and Desjardins [2013]. A generic mathematical description of an Euler-Lagrange information transfer with a spatial filtering is detailed in appendix B. In short, for the reconstruction of any Eulerian quantity $\phi$ at an

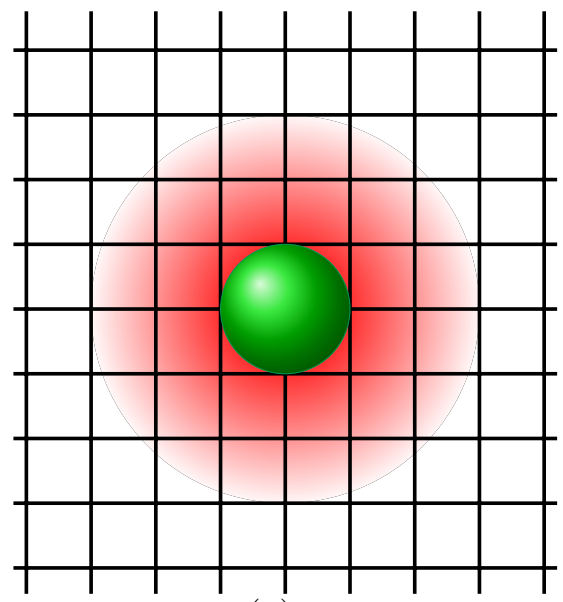

(a)

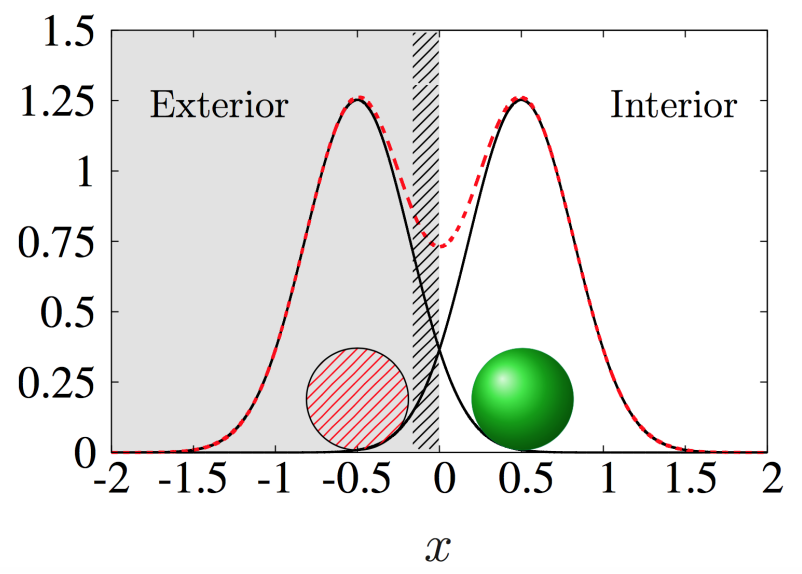

(b)

Figure 2. Gaussian Kernel averaging method: Illustration of (a) distribution of a particle property over the neighboring cells and (b) performance of the projection kernel close to a boundary. In (b), the real and image particles are in solid green, and red hashed pattern, respectively. The solid lines represent the projection kernel of the real and image particles, while the red dashed line is the superposition of both kernels. 


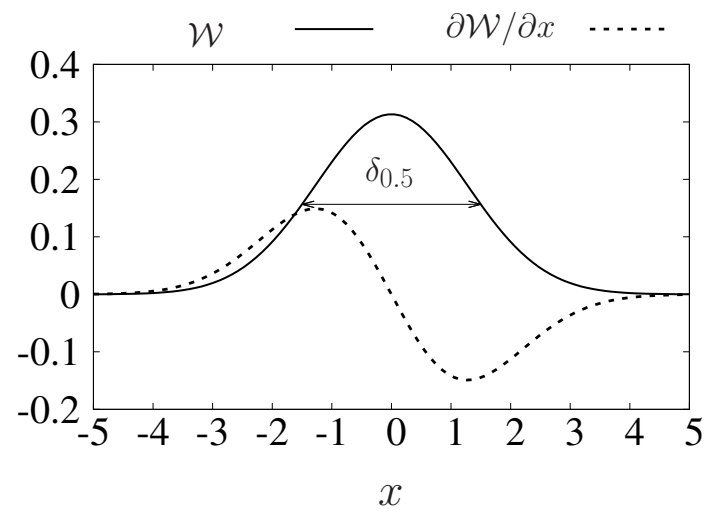

Figure 3. A Gaussian filtering kernel and its derivative. $\delta_{0.5}$ denotes the width of the kernel where it reaches $50 \%$ of its maximum value.

arbitrary Lagrangian position $\boldsymbol{r}_{i}$ we have:

$$
\phi\left(\boldsymbol{r}_{i}\right)=\frac{\int_{\Omega} \phi(\boldsymbol{x}) \mathcal{W}\left(\boldsymbol{x}-\boldsymbol{r}_{i}\right) d \boldsymbol{x}}{\int_{\Omega} \mathcal{W}\left(\boldsymbol{x}-\boldsymbol{r}_{i}\right) d \boldsymbol{x}}
$$

Where $\mathcal{W}$ denotes a symmetric kernel function centered at the Lagrangian position $\boldsymbol{r}_{i}$. For the reverse operation, viz. projecting particles information on the Eulerian grid reads as follows:

$$
\phi\left(\boldsymbol{x}_{j}\right)=\frac{\sum_{i=1}^{N_{p}} \phi\left(\boldsymbol{r}_{i}\right) \mathcal{W}\left(\boldsymbol{r}_{i}-\boldsymbol{x}_{j}\right) V_{p, i}}{\int_{\Omega} \mathcal{W}\left(\boldsymbol{x}-\boldsymbol{x}_{j}\right) d \boldsymbol{x}}
$$

While $V_{p, i}$ denotes the volume of particle $i$. A 3D Gaussian kernel centered at the origin reads as follows:

$$
\begin{aligned}
& \mathcal{W}(\boldsymbol{x})=\frac{\exp \left(-\boldsymbol{x}^{2} / 2 \sigma_{k}^{2}\right)}{\left(\sigma_{k} \sqrt{2 \pi}\right)^{3}}, \\
& \sigma_{k}=\delta_{0.5} / 2 \sqrt{2 \ln 2}
\end{aligned}
$$

As shown in Fig. 3, $\delta_{0.5}$ denotes the width where the kernel reaches $50 \%$ of its maximum value. Although the shape of the kernel may impact the smoothing properties and the numerical performance of the projection, we rely on a 
Gaussian function and do not further analyze the influence of the kernel shape on projection properties. For the Euler $\rightarrow$ Lagrange information transfer, the method implicitly handles the truncation of the domain by a simple normalization (c.f. equation 17, for instance). Hence, the interpolated values near the boundaries are less accurate, yet conservative. For the reverse operation Lagrange $\rightarrow$ Euler, we employ the mirror method suggested by Capecelatro and Desjardins [2013]. For the particles close to the wall, the truncated part of the projection kernel is redistributed over the interior domain in a conservative fashion as shown in FIG. 2(b). We consider an image particle in symmetry with the respective wall for all near-wall particles. The kernel for a near-wall particle $\mathcal{W}_{n w}$ is hence defined as the superposition of the original kernel and the kernel corresponding to the respective image particle:

$$
\mathcal{W}_{n w}\left(\boldsymbol{x}-\boldsymbol{r}_{i}\right)=\mathcal{W}\left(\boldsymbol{x}-\boldsymbol{r}_{i}\right)+\mathcal{W}\left(\boldsymbol{x}-\boldsymbol{r}_{i}^{i m g}\right)
$$

Where $\boldsymbol{r}_{i}^{i m g}$ is the position vector of the image particle. This simple method not only guarantees mass-conservation of the Euler $\rightarrow$ Lagrange projection step, but also enforces a Neumann boundary condition for the particle variables (c.f. FIG. 2(b)). While the true choice of the boundary condition for particle variables is the subject of many studies particularly in TFM approaches Benyahia et al. [2005]Li et al. [2010]Jenkins and Louge [1997], a Neumann boundary condition avoids the over-prediction of fluid volume fraction near walls as claimed by Capecelatro and Desjardins [2013]. According to our PRS numerical experiments, a slight increase of fluid volume fraction near walls is physical and related to the local geometric constraint. However, a non-conservative treatment of the projection operation over-predicts this increase and results in an unphysical behavior of the flow. Generally, a Neumann boundary condition along with a mass-conserving projection operation is deemed to properly pre- 
dict the flow behavior as well as the slight increase of near-wall fluid volume fraction.

\section{Simulation parameters}

The main parameters controlling the macroscopic properties of a fluidized bed are: (i) particle/fluid density ratio $\rho_{r}$, (ii) inlet flow Reynolds number $\mathcal{R} e_{i n}$, (iii) relative size of the particles with respect to the domain size and (iv) boundary conditions. In this study, we primarily target a smooth homogeneous fluidization with a relatively small system size. Accordingly, we choose a bi-periodic fluidization configuration with 512 particles. From now on, we choose the particle diameter $d^{*}$ and the inlet velocity $u_{i n}^{*}$ as characteristic length $L_{c}^{*}$ and velocity $V_{c}^{*}$, respectively, i.e., $L_{c}^{*}=d^{*}$ and $V_{c}^{*}=u_{i n}^{*}$. As illustrated in FIG. 4(a), the inlet flow is uniformly injected at $z=0$, while a constant pressure is imposed at the outlet boundary at $z=15$. The motivation behind this choice is that the homogeneity of the system allows a coherent statistical analysis in a relatively small domain. By choosing a density ratio

corresponding to a liquid/solid regime, we have spanned a limited range of $\mathcal{R} e_{i n}$ and $\varepsilon$.

Additionally, a heterogeneous fluidization in a high density ratio system is considered. Due to the larger length scales of the system, a larger domain is chosen: 2000 particles in a bi-periodic domain. Limited by the computational cost of this configuration, we have only chosen one set of parameters. The purpose of this simulation is mostly to compare for a critical test case, the performance of meso-scale models with a classical drag law as compared to PRS in a high density ratio regime. 
Assuming uniform axial inlet velocity and an a priori estimate of $\varepsilon_{m f}$, the minimum fluidization velocity for a homogeneous pack of spherical particles is derived from Gidaspow [1994]:

$$
\left(1-\varepsilon_{m f}\right)\left(\rho_{f}^{*}-\rho_{s}^{*}\right) \boldsymbol{g}^{*}=\beta \boldsymbol{u}_{m f}^{*}
$$

Where $\varepsilon_{m f}$ and $\boldsymbol{u}_{m f}^{*}$ denote the fluid volume fraction of the system and the inlet fluid velocity at the onset of fluidization, respectively. $\beta$ is the famous Ergun friction coefficient for a dense pack of spherical particles (Ergun [1952]). In all cases, we made sure that the inlet velocity is much larger than the minimum fluidization velocity.

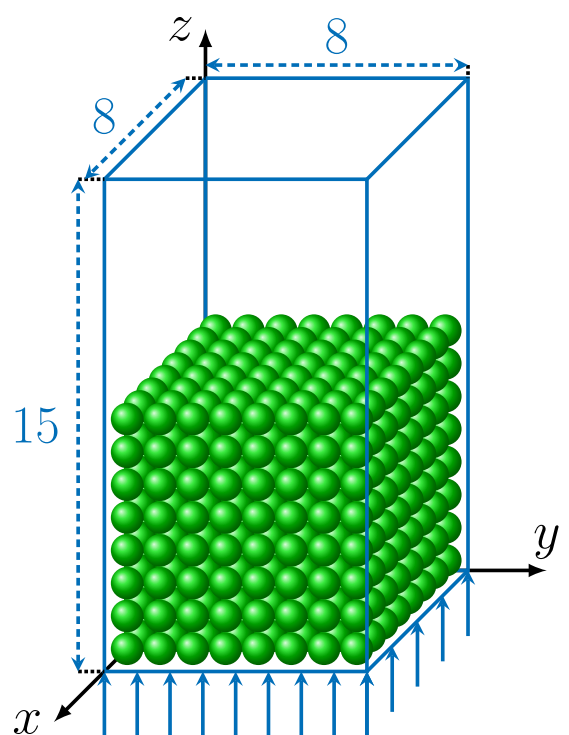

(a)

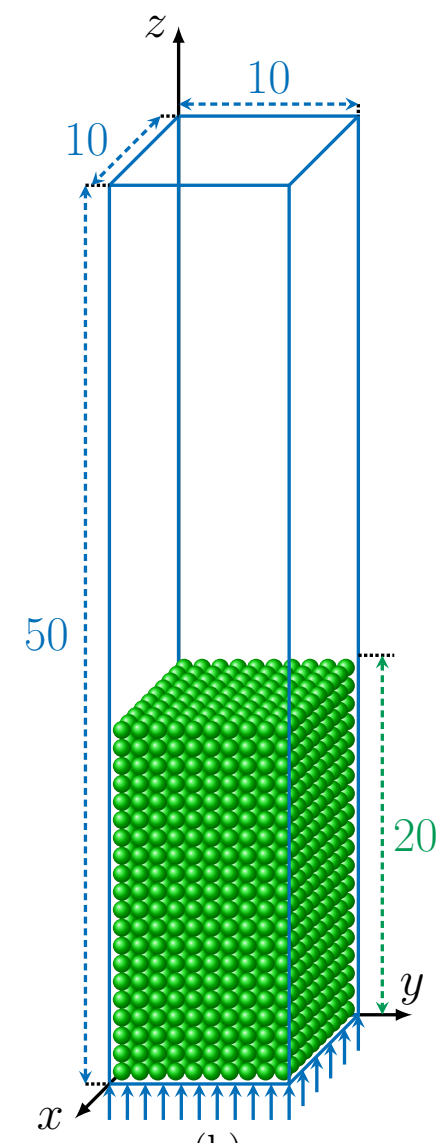

(b)

Figure 4. Flow domain and layout of particles at $t=0$ in (a) liquid/solid configurations and (b) gas/solid configurations. 
4 A stochastic formulation for the drag force

Table 1

Physical parameters of the system

\begin{tabular}{lcccccc}
\hline Case & $\mathcal{R} e_{p, i n}$ & $\mathcal{F} r$ & $\mathcal{S} t k$ & $\rho_{r}$ & $u_{i n, z}^{*} / u_{m f, z}^{*}$ & $N_{p}$ \\
\hline Liquid/solid & $3-11$ & $3.2-24.5$ & $1.7-6.2$ & 10 & $2-5.5$ & 512 \\
Gas/solid & 25 & 0.49 & 118 & 85 & 2 & 2000 \\
\hline
\end{tabular}




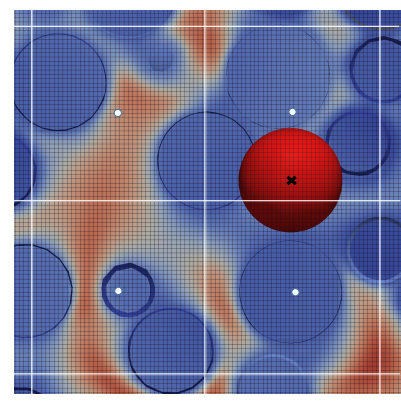

(a)

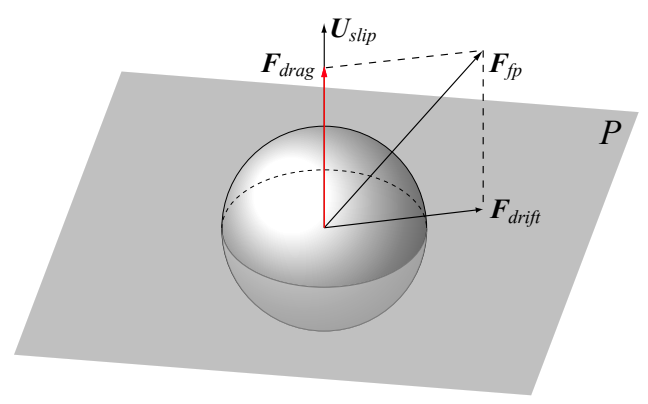

(b)

Figure 5. (a) Reconstruction of the PRS fluid field (black cells) at the DEM-CFD grid cell centers (white cells/points). Volume-averaged values are then linearly interpolated at the particle center. (b) Decomposition of the hydrodynamic force acting on a particle. The plane $P$ is perpendicular to the slip velocity vector $\left(\boldsymbol{U}_{\text {slip }}=\boldsymbol{u}-\boldsymbol{U}\right)$. $\boldsymbol{F}_{f p}$ denotes the total hydrodynamic force, $\boldsymbol{F}_{d r a g}$ and $\boldsymbol{F}_{d r i f t}$ are the components in parallel and perpendicular directions with respect to the slip velocity vector.

In this section, we first shortly present the post-processing and analysis of drag force acting on particles based on the PRS results. Next, we focus on the time-behavior of the drag force exerted on particles. These two steps serve as a basis for the description of our stochastic drag force, which will be subsequently explained. Statistical mean and standard deviation are denoted as $<$. $>$ and $\sigma($.$) , respectively, and defined for a quantity \phi$ as follows:

$$
\begin{aligned}
<\phi> & =\frac{1}{N_{\text {data }}} \sum_{i=1}^{N_{\text {data }}} \phi_{i} \\
\sigma_{\phi} & =\sqrt{\frac{1}{N_{\text {data }}} \sum_{i=1}^{N_{\text {data }}}\left(\phi_{i}-<\phi>\right)^{2}}
\end{aligned}
$$

where $N_{d a t a}$ is the number of data $\phi_{i}$ in the sample.

\subsection{Drag coefficient mean values and fluctuations}

Here, we average the PRS fluid field on the equivalent DEM-CFD grid cells with a simple box-hat filter $g(\boldsymbol{x})=1$, as shown in Fig. 5(a). For an Eulerian PRS quantity $\phi$, its average value denoted $\bar{\phi}$ in a DEM-CFD grid cell of volume 
$V_{D E M-C F D}$ is thereby computed as follows:

$$
\bar{\phi}=\frac{\int_{V_{D E M-C F D}} \chi(\boldsymbol{x}) g(\boldsymbol{x}) \phi(\boldsymbol{x}) d \boldsymbol{x}}{\int_{V_{D E M-C F D}} \chi(\boldsymbol{x}) g(\boldsymbol{x}) d \boldsymbol{x}}
$$

where $\chi(\boldsymbol{x})$ is a binary indicator function that is 1 in the fluid and 0 in any particle. Fluid Eulerian averaged variables are then interpolated at the particles position. In order to compute the drag coefficient of a particle, we estimate local porosity and fluid velocity at the particle position using the 2-step procedure (averaging followed by interpolating) described above. Such a filtering procedure provides us with an estimation of local fluid variables from PRS results which is most similar to its counterpart in a DEM-CFD simulation. Once the local fluid velocity around each particle is estimated, we can identify the direction of slip velocity and hence of drag force. As shown in FIG. 5(b), the drag force magnitude is estimated by projecting the PRS-based fluid particle force, $\boldsymbol{F}_{f p}$, on the slip velocity vector. The PRS drag coefficient for each particle is hence estimated as:

$$
\begin{aligned}
& C_{d}\left(\mathcal{R} e_{p}, \varepsilon\right)=\frac{8 \boldsymbol{F}_{d r a g}^{*}}{\pi \rho_{f}^{*} d^{* 2} \varepsilon^{2}\left(\boldsymbol{u}^{*}-\boldsymbol{U}^{*}\right)\left|\boldsymbol{u}^{*}-\boldsymbol{U}^{*}\right|} \\
& \mathcal{R} e_{p}=\frac{\rho_{f}^{*}\left|\boldsymbol{u}^{*}-\boldsymbol{U}^{*}\right| \varepsilon d^{*}}{\mu^{*}}
\end{aligned}
$$

The drag coefficient is considered to be a function of $\mathcal{R} e_{p}$ and $\varepsilon$ following the classical formulation of the drag law Ergun [1952]. Consequently, the drag coefficient experienced by each particle is instantaneously estimated. One might legitimately argue that the drag coefficient estimated by the instantaneous locally-averaged fluid field is different from the classical drag laws in two ways: a classical drag law (i) is derived in a stationary state e.g. flow past a fixed assembly of particles, and (ii) is based on far-field/integral properties e.g. pressure drop through the bed, inlet velocity, global fluid volume fraction. 
About (i), our interpretation of drag is in coherence with the DEM-CFD basic assumption, i.e., the mean drag considers local stationarity (Fan and Zhu [2005]). In that sense, a meso-scale model assumes that the particles can be considered to be in a quasi-steady state at each instant of time where there is no acceleration of the relative velocity ${ }^{1}$. Concerning (ii), the presence of mesoscale structures in a fluidized bed prevent us from employing far-field/integral properties to estimate the drag coefficient. Furthermore, since the meso-scale model also employs an equivalent compact local averaging kernel, our treatment of PRS results is consistent with the meso-scale model in a multi-scale framework. Finally, a drag coefficient based on locally-averaged variables provides us with the dispersion of drag coefficient for a given pair of $\mathcal{R} e_{p}$ and $\varepsilon$ in a fluidized state. Since our filtering operation is local, variations of the drag force from one realization to another for a given $\left(\mathcal{R} e_{p}, \varepsilon\right)$ pair is related to sub-filter scale fluctuations and local pore structures, rather than large-scale convective gradients. This supplementary information plays a key role in our stochastic description of the drag force.

Snapshots of the PRS field are collected at time intervals which are larger than the longest auto-correlation time of particles motion. Consequently, the drag coefficient corresponding to each particle at a given snapshot can be considered as a statistically independent realization. The drag coefficient is estimated for each particle and tabulated for a set of $\left(\mathcal{R} e_{p}, \varepsilon\right)$ pairs with an uncertainty tolerance of $1 \%$. FIG. 6 shows two examples of drag coefficient estimated by PRS and compared to the drag laws proposed in the literature for a given pair of $\left(\mathcal{R} e_{p}, \varepsilon\right)$. The drag coefficient predicted by PRS forms a

1 In fact, unsteady contributions of the relative velocity to the fluid/particle interaction force are lumped into added mass effects and Basset force which are virtually insignificant for large particle-fluid density ratios/non-oscillating flows (Fan and Zhu [2005]). 


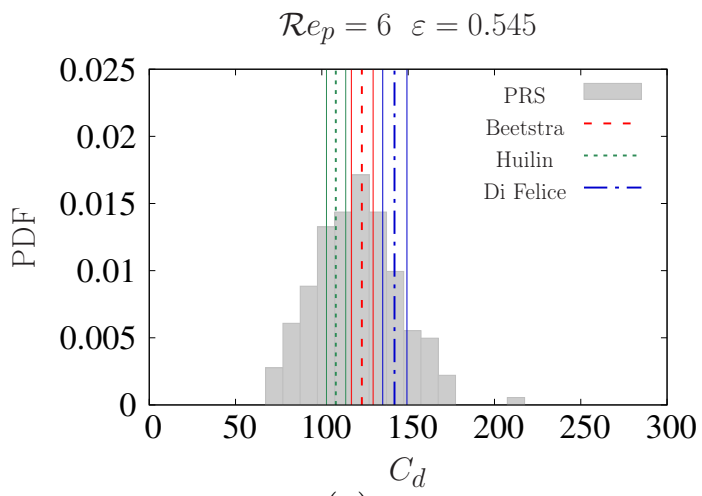

(a)

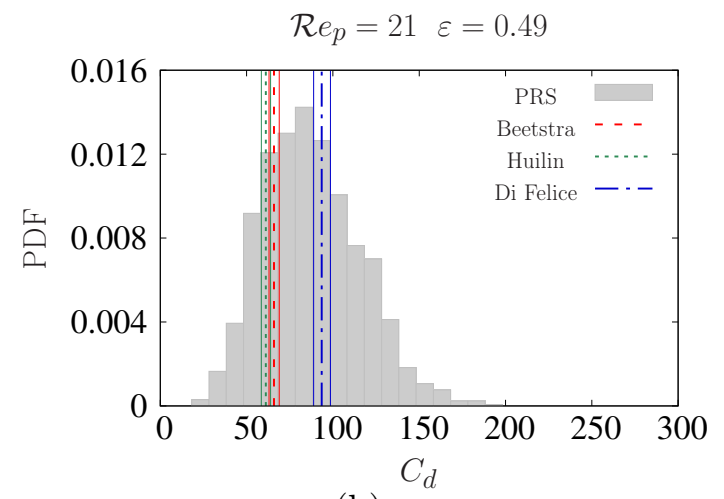

(b)

Figure 6. Probability Density Function (PDF) of drag coefficient, $C_{d}$, for a given pair of $\mathcal{R} e_{p}$ and $\varepsilon$ computed from the PRS results and different drag laws (Beetstra et al. [2007], Huilin and Gidaspow [2003], Di Felice [1994]) in (a) liquid/solid regimes and (b) gas/solid regimes. PRS results are shown as a PDF, and the deterministic drag correlations are shown by a Dirac function. The uncertainty of the values computed by the drag correlations are shown by solid lines and corresponds to the relative uncertainty tolerance of $1 \%$ in $\mathcal{R} e_{p}$ and $\varepsilon$.

distribution with a rather large dispersion of the data. To shed more light on the origins of these variations we investigate their dependence on the local values of $R e_{p}$ and $\varepsilon$. Before further detailing the characteristics of these PDFs, it is noteworthy to mention that as a general trend PDFs in the liquid/solid regime tend to a quasi-normal distribution, while they are mildly skewed toward larger values in the gas/solid regimes. The skewness is even more evident when considering the non-normalized absolute drag force (c.f. FIG. 11). In general, the convective gradients and heterogeneous meso-scale structures are responsible for such asymmetries.

We analyze the statistical dependence of the drag coefficient PDF to the number of realizations $N_{\text {real }}\left(=N_{\text {data }}\right.$ in (23)-(24)). As shown in FigS. 7 (a) and $7(\mathrm{c})$, while an increase of $N_{\text {real }}$ smoothes the PDF curves, its general shape is only subject to mild variations from 200 and 800 realizations in the liquid/solid and gas/solid regimes, respectively. Variations of the mean and standard deviation values with the number of realizations are shown in FiGs. 7(b) and 7(d). 


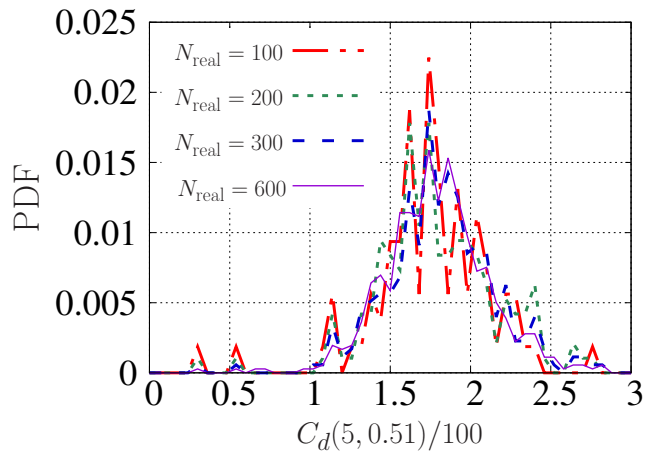

(a)

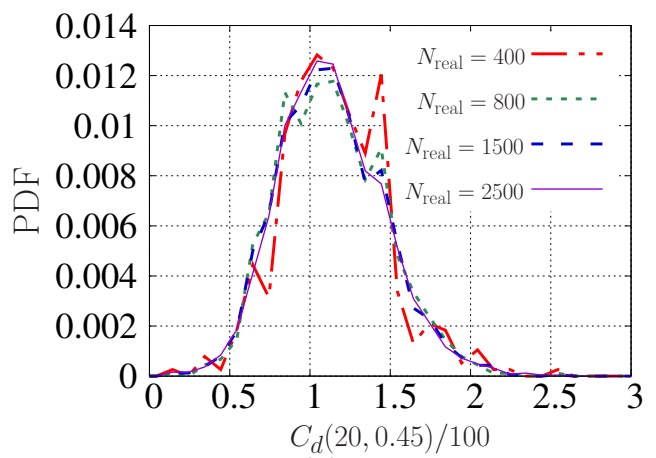

(c)

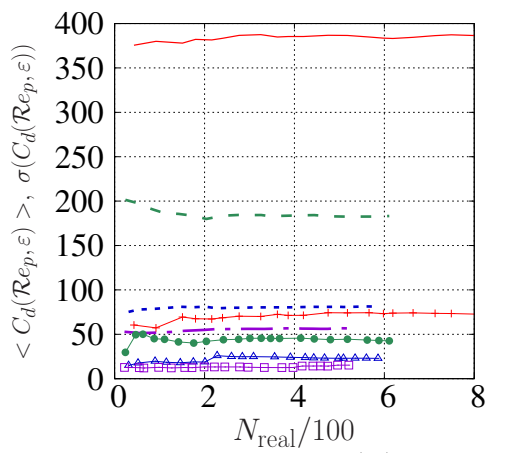

(b)

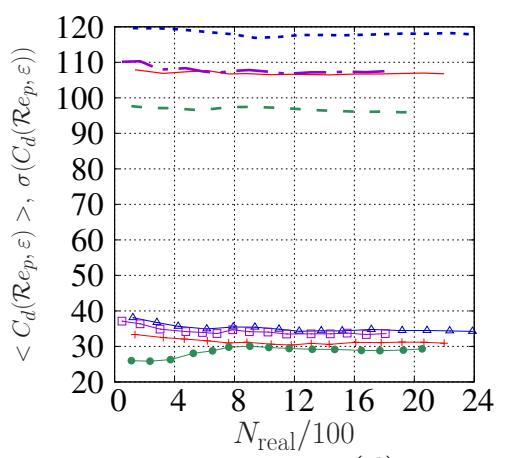

$<C_{d}(22,0.45)>$

$<C_{d}(22,0.47)>-$ - -

$<C_{d}(20,0.45)>$ - . . .

$<C_{d}(20,0.47)>-$ -

$\sigma\left(C_{d}(22,0.45)\right)$

$\sigma\left(C_{d}(22,0.47)\right) \longrightarrow$

$\sigma\left(C_{d}(20,0.45)\right) \triangle$

$\sigma\left(C_{d}(20,0.47)\right) \square$

Figure 7. Variation of the drag coefficient PDF for a given pair of $\mathcal{R} e_{p}$ and $\varepsilon$ with respect to the number of realizations in (a) liquid/solid regimes and (c) gas/solid regimes. (b) and (d) show the dependence of the mean and standard deviation values to the number of realizations, in liquid/solid regimes and gas/solid regimes respectively.

While mean values converge rapidly with number of realizations, a safe criterion to obtain less than $5 \%$ uncertainty range for standard variation values is $N_{\text {real }}=200$ and $N_{\text {real }}=800$, in the liquid/solid and gas/solid regimes, respectively. It should be noted that the number of realizations for a given $\left(\mathcal{R} e_{p}, \varepsilon\right)$ pair is not only limited by the simulation time, but also by the values of $\mathcal{R} e_{p}$ and $\varepsilon$ actually experienced by particles. Hence, the curves for different $\left(\mathcal{R} e_{p}, \varepsilon\right)$ pairs in Figs. $7(\mathrm{~b})$ and $7(\mathrm{~d})$ are truncated at different numbers of realizations for an equivalent simulation time, since the particles experience more frequently a certain $\left(\mathcal{R} e_{p}, \varepsilon\right)$ pair as compared to another.

Starting from mean drag, FIG. 8 shows PRS results compared to the drag law proposed by Beetstra et al. [2007]. Please note that in a fluidization con- 
figuration with a fixed density ratio, $\mathcal{R} e_{p}$ and $\varepsilon$ are interdependent. Hence, our PRS results do not show the decoupled functional dependence of $C_{d}$ on $\mathcal{R} e_{p}$ and $\varepsilon$. However, for each pair of $\left(\mathcal{R} e_{p}, \varepsilon\right), C_{d}^{\text {PRS }}$ fits well $C_{d}^{\text {correl }}$ as shown in FIGS. 8(a) and 8(b). It is surprising to us that these relatively old drag laws - which are by the way widely used in meso-scale models - fit better as compared to new highly-resolved drag laws proposed in the literature (Tenneti et al. [2011],Tang et al. [2015],Bogner et al. [2015]). As pointed out by Tang et al. [2016], the drag force experienced by moving particles is by nature larger. We also think that the fact that most of these drag laws are based on static particle arrangements explains this seeming inconsistency. Recently, Tang et al. [2016] proposed a drag law for moving particles which also takes into account the granular temperature of the particles. However, our set of parameters is not in its range of validity. For the aforementioned reasons, we have intentionally avoided to quantitatively compare different drag laws with $C_{d}^{\mathrm{PRS}}$. Once again, the main purpose of this study is to investigate the influence of drag force fluctuations. As we will see later in Section 5, incorporating drag laws with matching mean values as compared to PRS result in good predictions on integral properties, yet underprediction of particles velocity fluctuations.

Now we turn our attention to any possible correlation between standard deviation of drag coefficient and local porosity and Reynolds number. An increasing trend between dispersion of drag coefficient and porosity of the system has already been reported in the literature in the simulation of flow past random arrays of fixed particles. According to Beetstra et al. [2007], in a homogeneous fixed bed configuration the drag force applied to an individual particle commonly varies by a factor of 2 to 3 , while dispersion increases with fluid volume fraction. We investigate the sensitivity of $\sigma\left(C_{d}^{\mathrm{PRS}}\right)$ to the variation 
of $\mathcal{R} e_{p}$ and $\varepsilon$. The standard deviation of drag coefficient normalized by the drag coefficient mean value increases with an increase in $\mathcal{R} e_{p}$ and $\varepsilon$ in both regimes. Since these two dimensionless parameters are interdependent in our system, we study the correlation between $\sigma\left(C_{d}^{\mathrm{PRS}}\right)$ and $\mathcal{R} e_{p}$ only. As shown in FIG. 10, similar to the functional dependence of mean drag to $R e_{p}$, a clear negative correlation between $\sigma\left(C_{d}^{\mathrm{PRS}}\right)$ and $\mathcal{R} e_{p}$ is present in both regimes. We employ a power law and a linear fit in order to quantify the correlation, while

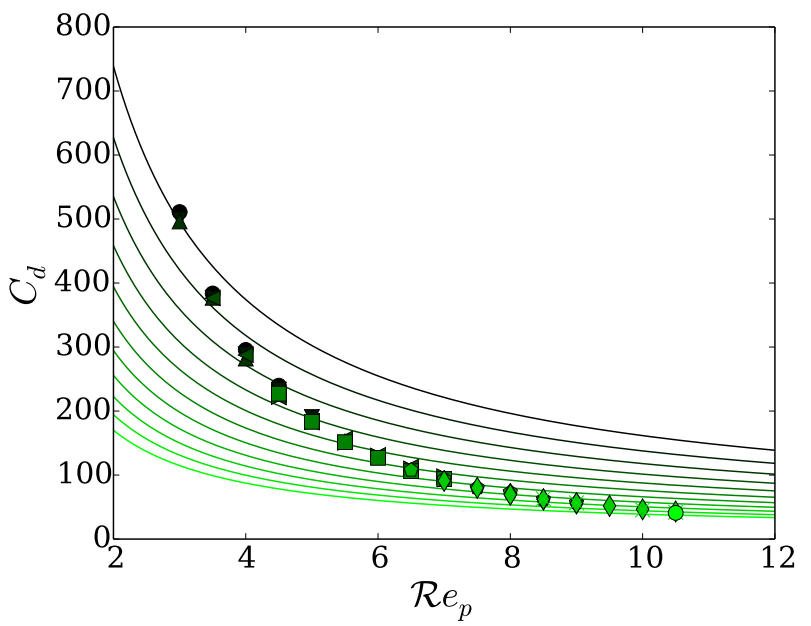

\begin{tabular}{|c|c|c|}
\hline$-C_{d}^{\text {correl }}\left(\mathcal{R} e_{p}, 0.45\right)$ & $\bullet$ & $<C_{d}^{\mathrm{PRS}}\left(\mathcal{R} e_{p}, 0.45\right)>$ \\
\hline$-C_{d}^{\text {correl }}\left(\mathcal{R} e_{p}, 0.47\right)$ & $\boldsymbol{\nabla}$ & $<C_{d}^{\mathrm{PRS}}\left(\mathcal{R} e_{p}, 0.47\right)>$ \\
\hline$C_{d}^{\text {correl }}\left(\mathcal{R} e_{p}, 0.49\right)$ & $\Delta$ & $<C_{d}^{\mathrm{PRS}}\left(\mathcal{R} e_{p}, 0.49\right)>$ \\
\hline$C_{d}^{\text {correl }}\left(\mathcal{R} e_{p}, 0.51\right)$ & 4 & $<C_{d}^{\mathrm{PRS}}\left(\mathcal{R} e_{p}, 0.51\right)>$ \\
\hline$C_{d}^{\text {correl }}\left(\mathcal{R} e_{p}, 0.53\right)$ & $\triangleright$ & $<C_{d}^{\mathrm{PRS}}\left(\mathcal{R} e_{p}, 0.53\right)>$ \\
\hline$C_{d}^{\text {correl }}\left(\mathcal{R} e_{p}, 0.55\right)$ & $\nabla$ & $<C_{d}^{\mathrm{PRS}}\left(\mathcal{R} e_{p}, 0.55\right)>$ \\
\hline$C_{d}^{\text {correl }}\left(\mathcal{R} e_{p}, 0.57\right)$ & + & $<C_{d}^{\mathrm{PRS}}\left(\mathcal{R} e_{p}, 0.57\right)>$ \\
\hline$C_{d}^{\text {correl }}\left(\mathcal{R} e_{p}, 0.59\right)$ & $\bullet$ & $<C_{d}^{\mathrm{PRS}}\left(\mathcal{R} e_{p}, 0.59\right)>$ \\
\hline$C_{d}^{\text {correl }}\left(\mathcal{R} e_{p}, 0.61\right)$ & $\diamond$ & $<C_{d}^{\mathrm{PRS}}\left(\mathcal{R} e_{p}, 0.61\right)>$ \\
\hline$C_{d}^{\text {correl }}\left(\mathcal{R} e_{p}, 0.63\right)$ & $x$ & $<C_{d}^{\mathrm{PRS}}\left(\mathcal{R} e_{p}, 0.63\right)>$ \\
\hline$-C_{d}^{\text {correl }}\left(\mathcal{R} e_{p}, 0.65\right)$ & 0 & $<C_{d}^{\mathrm{PRS}}\left(\mathcal{R} e_{p}, 0.65\right)>$ \\
\hline
\end{tabular}

(a)
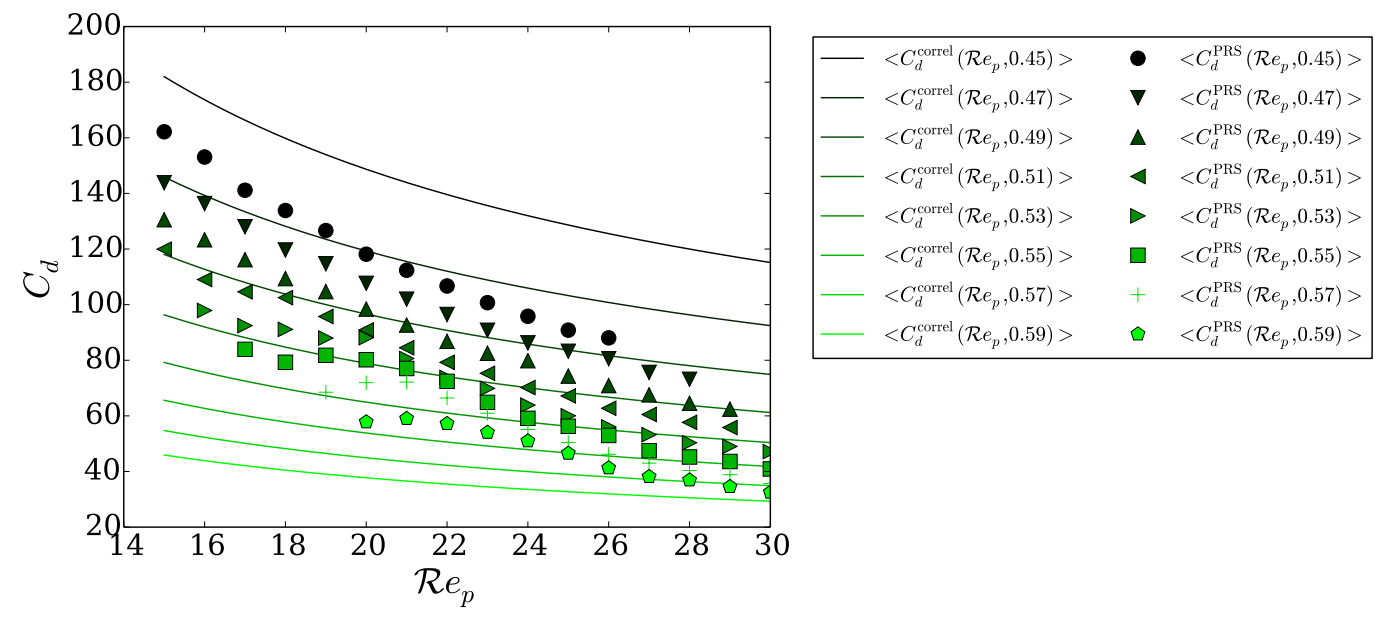

(b)

Figure 8. Drag coefficient as a function of $\mathcal{R} e_{p}$ and for different $\varepsilon$. Drag correlations $C_{d}^{\text {correl }}$ in the literature (Beetstra et al. [2007] and Di Felice [1994] for (a) liquid/solid regimes and (b) gas/solid regimes, respectively) and drag coefficients $C_{d}^{\mathrm{PRS}}$ predicted by PRS are represented by lines and symbols, respectively. 
we acknowledge that the graphical linearity of the trend in gas/solid regime is an artifact of the short $\mathcal{R} e_{p}$ range. However, recalling that the preceding fits are to be employed in DEM-CFD simulations in which particles presumably experience an equivalent range of $\mathcal{R} e_{p}$ as compared to PRS, such simplification is deemed to be acceptable. Indeed, a full description of the correlation between $\sigma\left(C_{d}^{\mathrm{PRS}}\right)$ and $\mathcal{R} e_{p}$ requires a parametric analysis by performing several PRS for a wide range of $\mathcal{R} e_{p}, \varepsilon$ and density ratio.

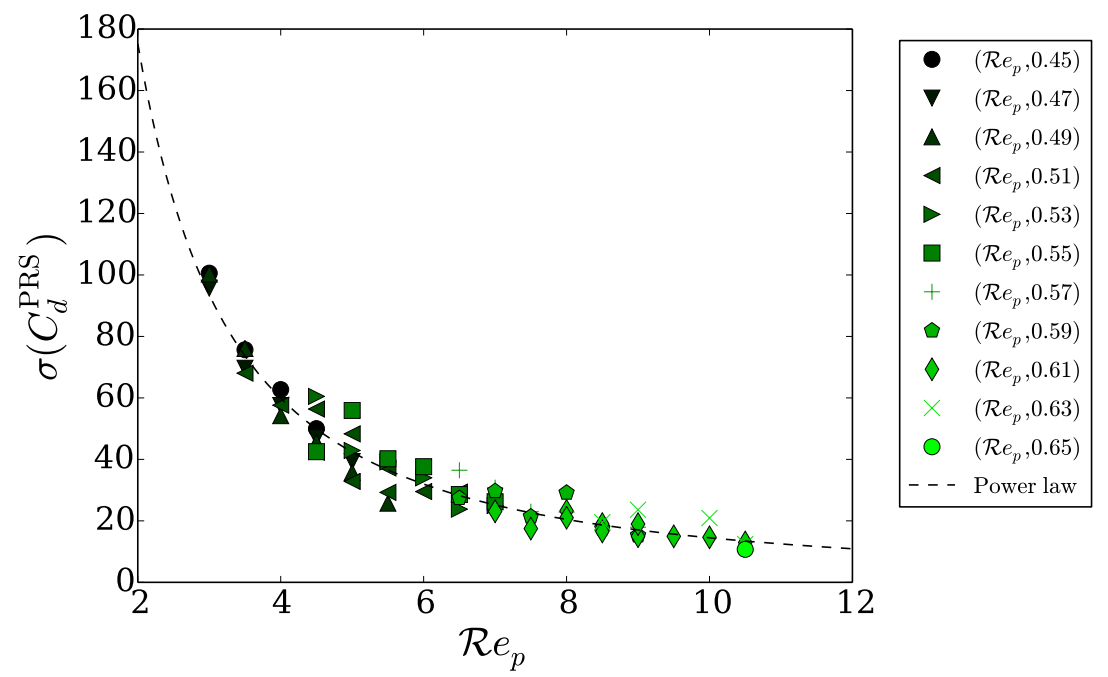

(a)

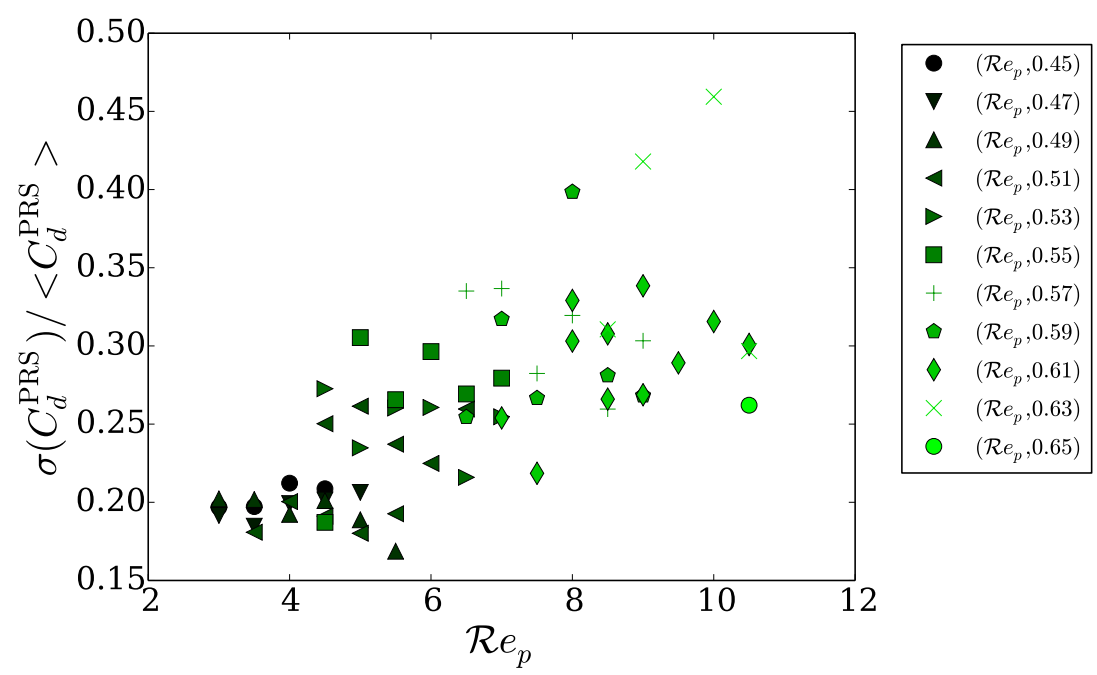

(b)

Figure 9. Standard deviation of the drag coefficient in liquid/solid regimes as a function of $\mathcal{R} e_{p}$ and for different $\varepsilon$ : (a) absolute value and (b) value normalized by mean drag coefficient. 


\subsection{Time behavior of fluctuations}

So far we have analyzed the drag force fluctuations based on snapshots of the PRS velocity field. In our approach PDFs are extracted from the ensemble of particles at different times: a combination of spatial and temporal fluctuations. These fluctuations which stem from sub-grid convective gradients, can be modeled by a stochastic process. Such treatment by definition assumes that

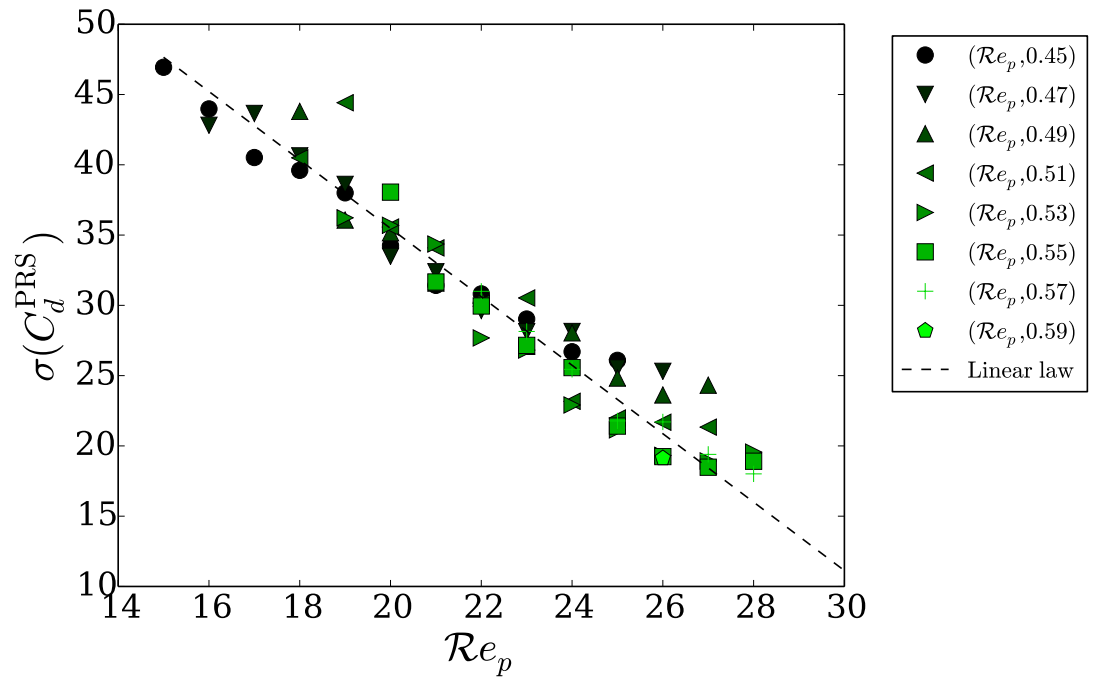

(a)

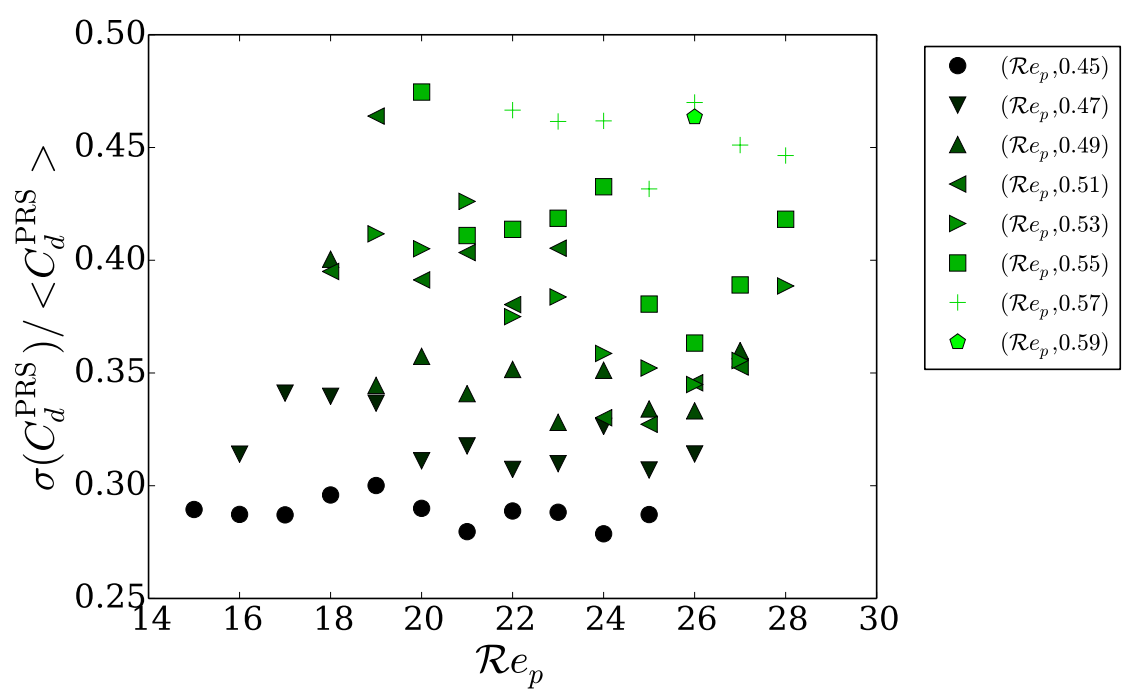

(b)

Figure 10. Standard deviation of the drag coefficient in the gas/solid regime as a function of $\mathcal{R} e_{p}$ and for different $\varepsilon$ : (a) absolute value and (b) value normalized by mean drag coefficient. 
the spatial and temporal behaviors of the drag force exerted on the particles are equivalent (ergodicity assumption). Thanks to PRS, we can readily verify to which extent this assumption is valid. FIG. 11 shows the distribution of the drag force magnitude over the ensemble of particles at an instant of time

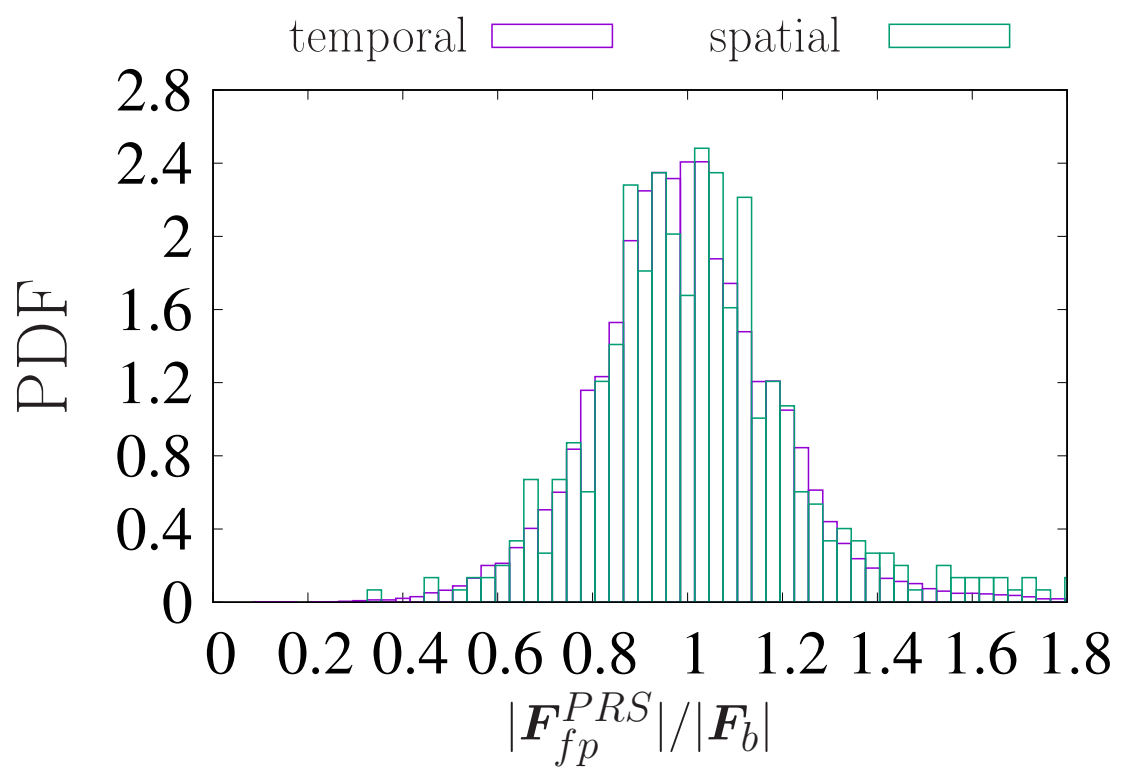

(a)

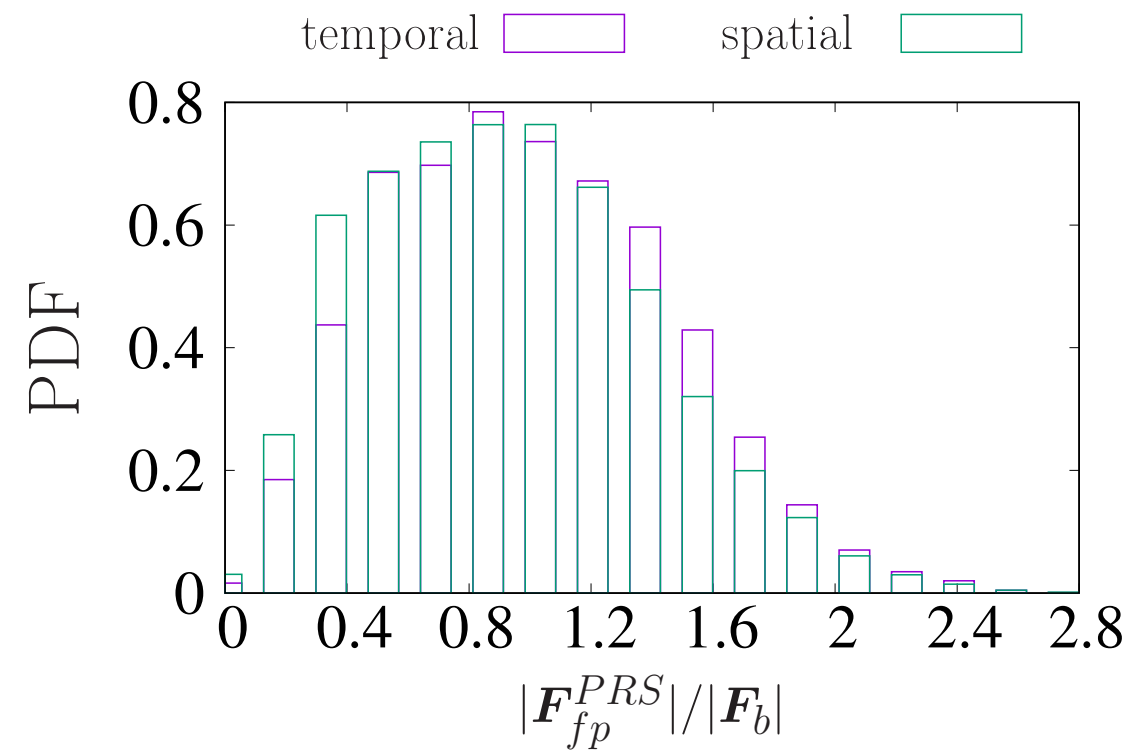

(b)

Figure 11. PDF of the fluid/particle force normalized by the buoyancy force over (a) ensemble of particles (spatial) or (b) particle's trajectory (temporal) in liquid/solid regimes. 
(spatial) and over a particle trajectory (temporal). First, differences between distributions in time and space are quite limited, emphasizing the validity of the ergodicity assumption. Second, distribution forms are quite different in the gas/solid and liquid/solid regimes. The former is close to normal, while the latter is clearly skewed toward larger values. Such asymmetries in drag force PDFs highlight the significance of large-scale motions in the gas/solid regime.

In order to have a complete picture of the drag force fluctuations, we study its

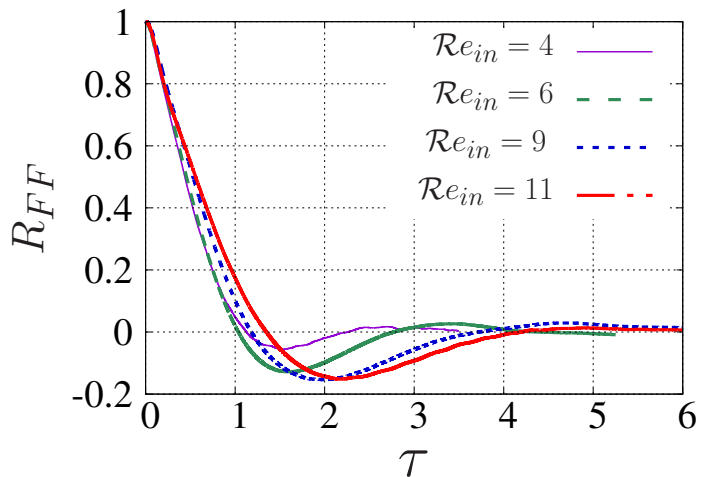

(a)

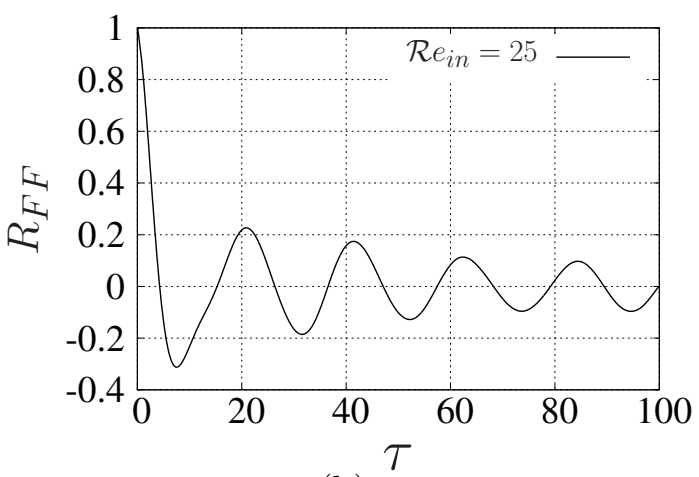

(b)

Figure 12. Auto-correlation function of the drag force magnitude as a function of the dimensionless separation time in (a) liquid/solid regimes and (b) gas/solid regimes.

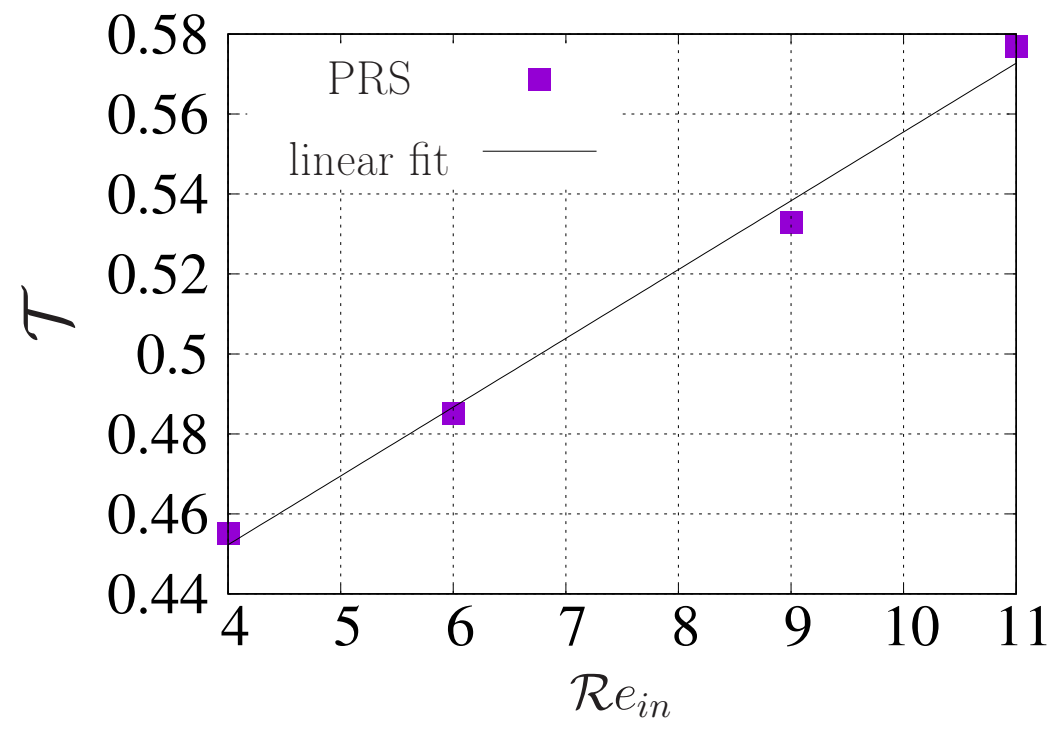

Figure 13. Correlation time $\mathcal{T}$ as a function of inlet Reynolds number in liquid/solid regimes. 
characteristic time. Although particles motion is indeed far from Brownian, we assume that it consists of some random modes representing the pseudothermal agitation of particles. This hypothesis can be further examined by studying the drag force auto-correlation function defined as:

$$
\begin{aligned}
& R_{F F}(\tau)=\frac{<F_{f p, i}^{\prime \prime}(t+\tau) F_{f p, i}^{\prime \prime}(t)>}{<F_{f p, i}^{\prime \prime}(t) F_{f p, i}^{\prime \prime}(t)>}, i=x, y, z \\
& F_{f p, i}^{\prime \prime}=F_{f p, i}-<F_{f p, i}>, \quad i=x, y, z
\end{aligned}
$$

FIG. 12 shows the auto-correlation function for the drag force magnitude in liquid/solid and gas/solid regimes. As expected, the co-existence of random motion at early separation times and large-scale convective motion at larger separation times are present in both regimes. Nevertheless, the convective motion in the form of a harmonic wave is obviously dominant in the gas/solid regime. The integral time of the auto-correlation function is classically defined as:

$$
\mathcal{T}=\int_{0}^{\tau_{\infty}} R_{F F}(\tau) d \tau
$$

Where $\tau_{\infty}$ denotes an infinitely large separation time. For a purely random motion, the autocorrelation function is expected to decay exponentially. In that hypothetical condition, the integral in equation 30 exponentially tends to the characteristic time of fluctuations. This is not the case in the presence of convective gradients. The integral in equation 30 as a function of the upperbound, $\tau_{\infty}$, oscillates around a steady level. We have simply chosen this steady level as the fluctuations characteristic time. Hence, in our particular analysis we re-define the equation 30 as:

$$
\mathcal{T}=<\int_{0}^{\tau_{\infty}} R_{F F}(\tau) d \tau>_{\tau_{\infty}}
$$

Where the averaging is performed over the quasi-steady separation time slot, namely $\tau_{\infty}>4$ and $\tau_{\infty}>40$ in the liquid/solid and gas/solid regimes, 
respectively. Although quite simple, our approach eliminates the influence of large-scale harmonic motions on the estimation of the pseudo-thermal fluctuations time. The fluctuations integral time varies with the dimensionless parameters of the system. In the liquid/solid regime, FIG. 13 shows the dependence of this value on $\mathcal{R} e_{i n}$. A linear fit is used to correlate the fluctuations integral time to $\mathcal{R} e_{i n}$ in the liquid/solid regime.

\subsection{Fluctuating drag as a stochastic event}

We have obtained a rather complete picture of the drag force fluctuations in the range of dimensionless parameters of our PRS. We have shown that despite some differences in the functional dependence of the mean $\operatorname{drag}$ on $\mathcal{R} e_{p}$ and $\varepsilon$, the average value for the given range of dimensionless parameters is almost in accordance with PRS. We now focus on a description of the drag coefficient which represents sub-grid fluctuations by a stochastic event, namely $C_{d}^{s}$ :

$$
C_{d}^{s}=\overline{C_{d}}+C_{d}^{\prime}
$$

Where $C_{d}^{\prime}$ and $\overline{C_{d}}$ represent the fluctuating and mean components, respectively. Recall that the term mean is defined with respect to sub-grid fluctuations and simply implies that $\overline{C_{d}}$ is a mere function of locally averaged variables. However, both $\overline{C_{d}}$ and $C_{d}^{\prime}$ are indeed time-dependent variables in a freely-moving particles system. We seek a stochastic signal with the following mathematical features: (i) its long-run mean should be zero, (ii) it should enjoy a smooth power spectrum without any preference toward a given frequency (iii) long-run variance and time-correlation should be easily adjustable. With that in mind and inspired by time-series analysis methods (Box et al. [2015]), 
we employ a simple first order Auto-Regressive (AR-1) signal:

$$
\begin{aligned}
& C_{d}^{\prime}(t+\Delta t)=a C_{d}^{\prime}(t)+b \sqrt{\left(1-a^{2}\right)} w(t) \\
& <w(t)>_{t}=0, \quad \sigma(w(t))=1
\end{aligned}
$$

Where $w(t)$ represents a Gaussian white noise with a zero mean and standard deviation of 1, a measures the extent to which the signal retains its memory, $b$ controls the intensity of the randomness and $\Delta t$ is the time-step. In fact, an AR-1 process can be considered as a discrete time equivalent of the continuous Ornstein-Uhlenbeck process. Its conditional expectancy and variance can hence be written in the following form:

$$
\begin{aligned}
& E\left[C_{d}^{\prime}(t+n \Delta t) \mid C_{d}^{\prime}(t)\right]=a^{n \Delta t} C_{d}^{\prime}(t) \\
& \operatorname{Var}\left[C_{d}^{\prime}(t+n \Delta t) \mid C_{d}^{\prime}(t)\right]=b^{2}\left(1-a^{2 n \Delta t}\right)
\end{aligned}
$$

Given that $|a|<1$, the long-run mean and variance of the signal tend to zero and $b^{2}$, respectively. The time-behavior of the signal described by its autocorrelation function is given as:

$$
R_{C_{d}^{\prime} C_{d}^{\prime}}(\tau)=\exp (-\tau / \mathcal{T}), \quad \mathcal{T}=-\Delta t / \ln a
$$

Hence, for a desired standard deviation $\sigma\left(C_{d}^{\prime}\right)$ and fluctuation integral time $\mathcal{T}^{\text {PRS }}$ estimated from PRS results and for a given $\Delta t$, the model parameters $a$ and $b$ are computed. FIG. 14 shows an example of the signal time evolution as well as its auto-correlation function. We have normalized the $x$-axis in FIG. 14(b) by the integral time corresponding to the respective curve in order to compare the shape of auto-correlation functions (c.f. (30) and (31) for definition of $\mathcal{T}) . C_{d}^{\text {det }}$ corresponds to a DEM-CFD simulation with a classical deterministic drag force without any fluctuating component, viz. $C_{d}^{\text {det }}=\overline{C_{d}}$. Likewise, $C_{d}^{s t o}$ and $C_{d}^{\prime}$ correspond to a stochastic drag coefficient and its fluctuating component in a DEM-CFD simulation incorporating a stochastic drag 
force. First, $C_{d}^{\prime}$ decays exponentially with the separation time, as expected for an $A R-1$ process. Furthermore, there is nearly no difference between the shape of the auto-correlation function corresponding to a classical drag coefficient without stochastic component, $C_{d}^{d e t}$, and the stochastic description of the drag coefficient, $C_{d}^{s t o}$. Thus, it is safe to conclude that the fluctuating term $C_{d}^{\prime}$ merely mimics the pseudo-thermal fluctuations and does not affect the convective motion of particles at large separation times.

\section{Results}

We intend to compare the performance of three variants of the meso-scale model: (i) a bounding cube method for interphase coupling and a classical deterministic drag law, (ii) a Gaussian kernel method for interphase coupling and a classical deterministic drag law, and (iii) a Gaussian kernel method for interphase coupling and the proposed stochastic drag law. Results produced by these three variants of meso-scale model are compared to that of PRS which

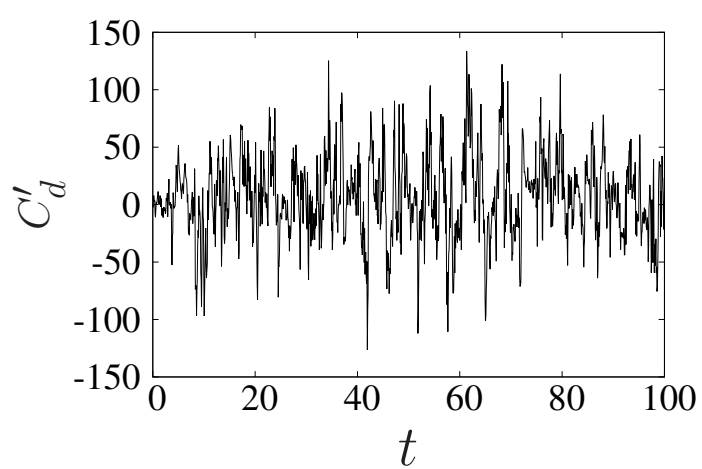

(a)

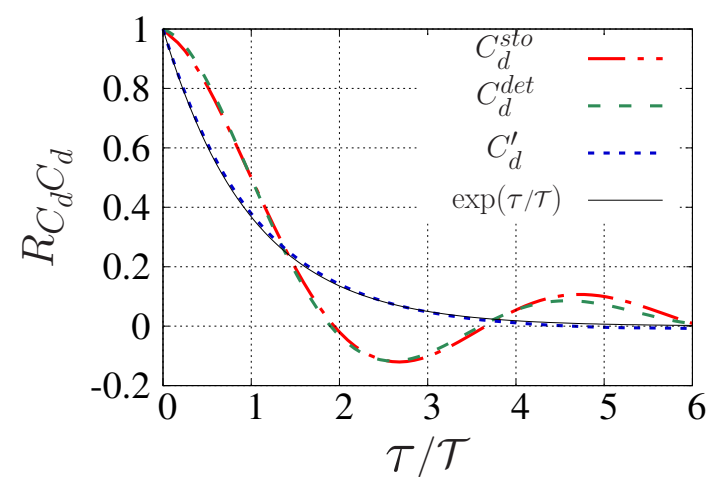

(b)

Figure 14. (a) An example of $C_{d}^{\prime}$ signal as a function of time and (b) autocorrelation function of a stochastic drag coefficient $C_{d}^{\text {sto }}$ and its fluctuating component, $C_{d}^{\prime}$ (c.f. (33)) compared to a classical drag coefficient without stochastic component, $C_{d}^{\text {det }}$. The $x$-axis is normalized by the integral time, $\mathcal{T}$ (defined in (30) and (31)), corresponding to the respective curve. Both plots correspond to a DEM-CFD simulation in a liquid/solid regime. 
serves as a reference. We start with the liquid/solid regime. Four cases with different inlet velocities are considered in the liquid/solid regime. Next, we turn our attention to the gas/solid regime. Numerical parameters are listed in TAB. 2. We introduce an abbreviation in referring to our DEM-CFD simulations: the two first letters denote the interphase coupling method (BC for the bounding cube and GK for the Gaussian kernel) and the following number specifies the grid resolution $\Delta x^{-1}$. The choice of the grid size in micro-scale simulations have been the subject of our previous study (Esteghamatian et al. [2017b]). For the meso-scale model, we performed a grid- and kernel support size-refinement analysis detailed in APPX. C. 
Table 2

Numerical parameters of DEM-CFD and PR simulations.

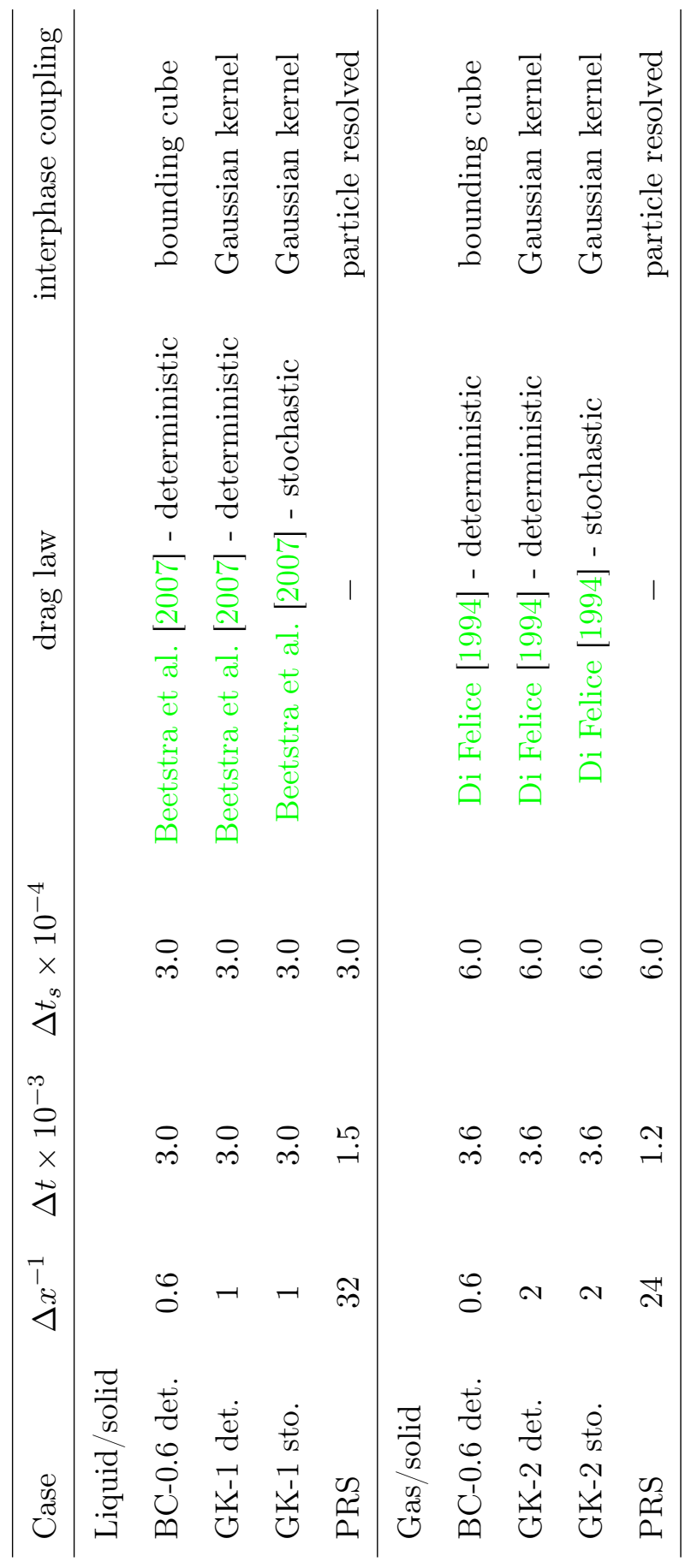

\subsection{Liquid/solid}

FIG. 15 compares the axial fluid volume fraction profile predicted by different models. Except for the very beginning and end of the bed, a smooth profile is 
predicted by PRS. In the two lowest $\mathcal{R} e_{i n}$ cases, BC-0.6 with a deterministic drag law predicts large variation along $z$-axis which is due to the presence of nonphysical particle structures. Using smaller grid size with Gaussian kernel method attenuates the presence of such structures. In our previous study with the same physical parameters in a slightly larger configuration and using the BC-0.6 method, such effects were almost absent (Esteghamatian et al. [2017a]). The bed height is almost well-predicted by all variants of the meso-scale model. In general, the bed height is an indicator of the accuracy of the mean drag coefficient. In line with our findings in Section 4.1, the mean drag coefficient predicted by PRS and the deterministic drag law proposed by Beetstra et al. [2007] are in agreement (see FIG. 8). Finally, there is virtually no difference between the results predicted by GK-1 det. and GK-1 sto., implying that the stochastic formulation for the drag has no effect on the mean drag force value.

FIG. 16 shows the time evolution of square root of particles granular temperature. First, using the GK method improves the prediction of the level of particles granular temperature as compared to PRS results. In spite of this improvement, the particle axial fluctuations are still not fully captured with a deterministic drag. The DEM-CFD simulations with the stochastic drag and the GK coupling method, however, correctly predict the particles fluctuation level within the uncertainty range of PRS results.

In the transverse direction as shown in FIG. 17, the general picture is similar with an improved prediction with GK-1 and stochastic drag. Nonetheless, the granular temperature is still slightly underpredicted as compared to PRS. In an effort to identify the origins of this discrepancy, we once again refer to PRS. In Esteghamatian et al. [2017a], PRS results revealed that the fluid/particle force exerted on a particle is not necessarily co-linear with respect to its slip velocity vector. As a result, the fluid/particle force has a perpendicular 
component with respect to the slip velocity. Although our proposed stochastic drag force already takes into account the drag force magnitude fluctuations, it neglects the fluctuating behavior of the drag force direction. We believe that the limited deviation of transverse particles granular temperature predicted by GK-1 and stochastic drag as compared to PRS is associated with the lack of a fluctuating force perpendicular to the slip velocity vector. Nevertheless, it should be noted that particles transverse motion is not exclusively driven by the fluid/particle force. In Esteghamatian et al. [2017b], we observed that collision is an efficient mechanism of momentum transfer from mean flow to transverse particle motion. Hence, the stochastic drag (which is parallel to the slip velocity and therefore is mostly oriented in the axial direction) is implicitly projected to the transverse directions via inter-particle colli-

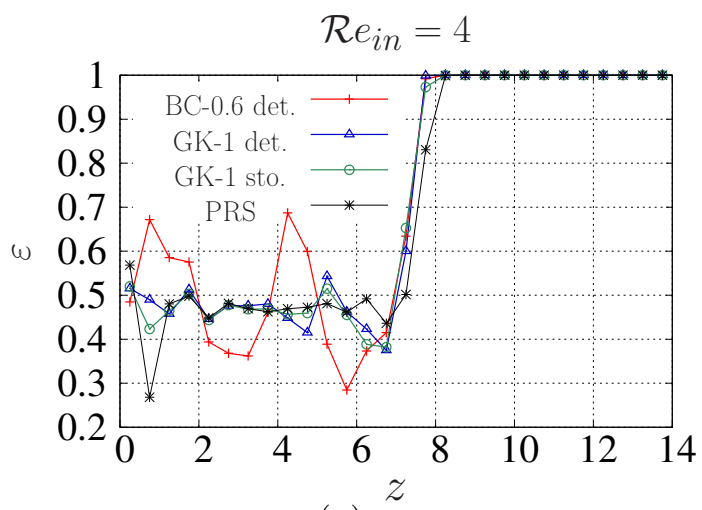

(a)

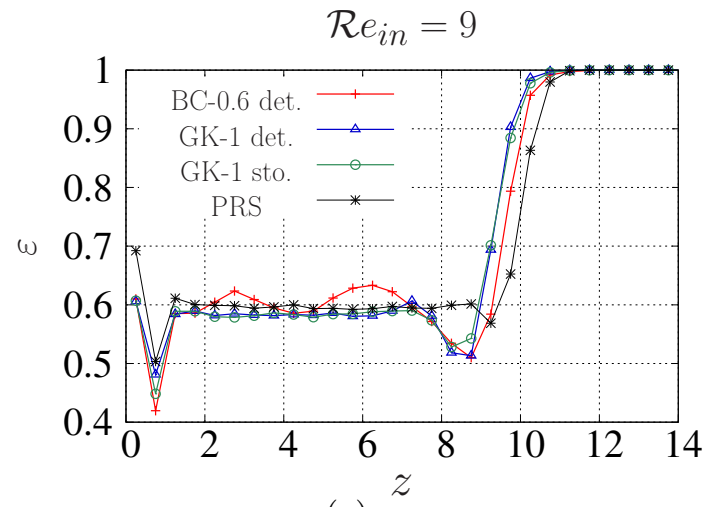

(c)

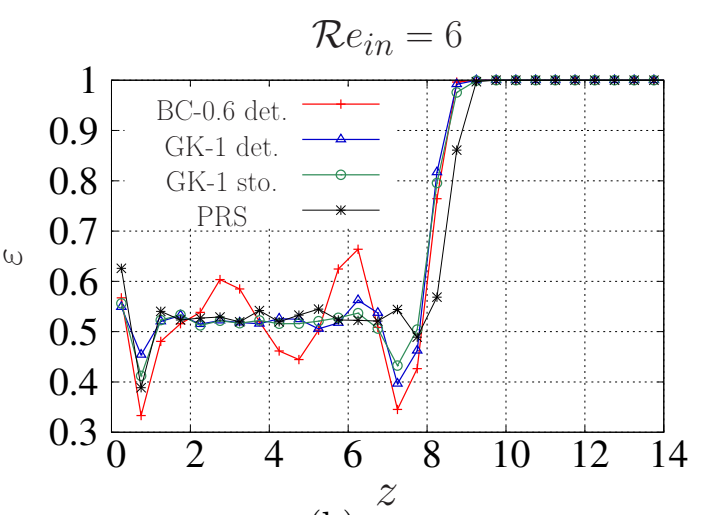

(b)

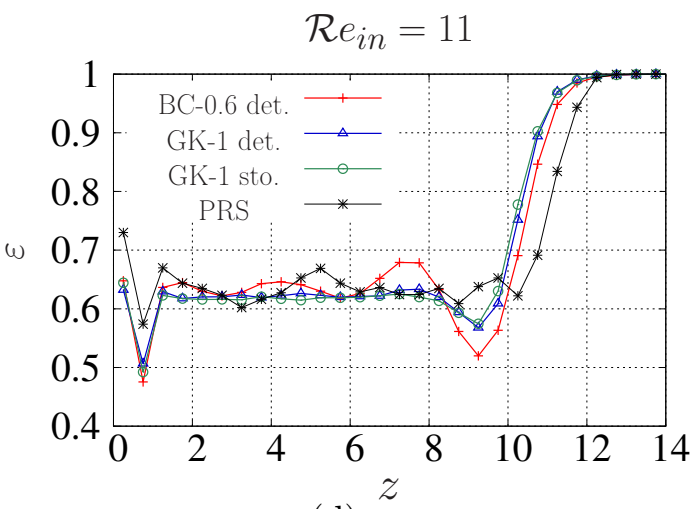

(d)

Figure 15. Fluid volume fraction axial profile in the liquid/solid regime with different inlet velocities. The definition of abbreviations is given in TAB. 2 . 
sion, leading to the improvement of particles transverse granular temperature.

\subsection{Gas/solid}

The type of fluidization in the gas/solid regime is pretty different from a hydrodynamic viewpoint as compared to the liquid/solid regime Esteghamatian et al. [2017b]. Based on PRS, we clearly observe the emergence, growth and burst of a bubble in a periodic fashion. FiG. 18 graphically shows the bed evolution and particle positions as well as fluid velocity contours predicted by PRS and DEM-CFD simulations. The overall picture is surprisingly quite

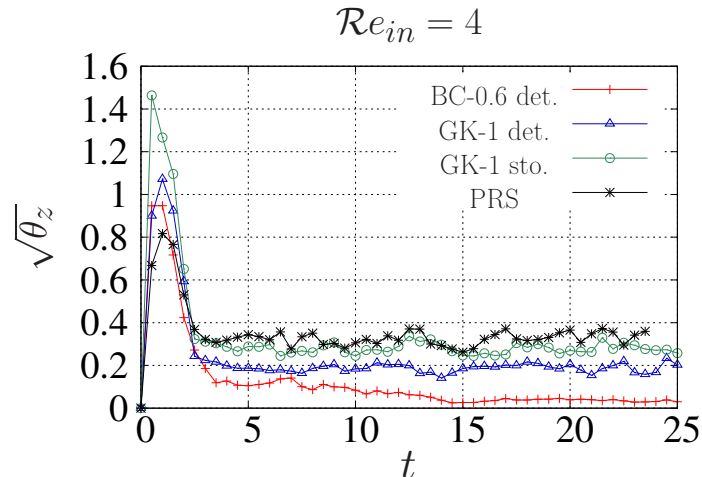

(a)

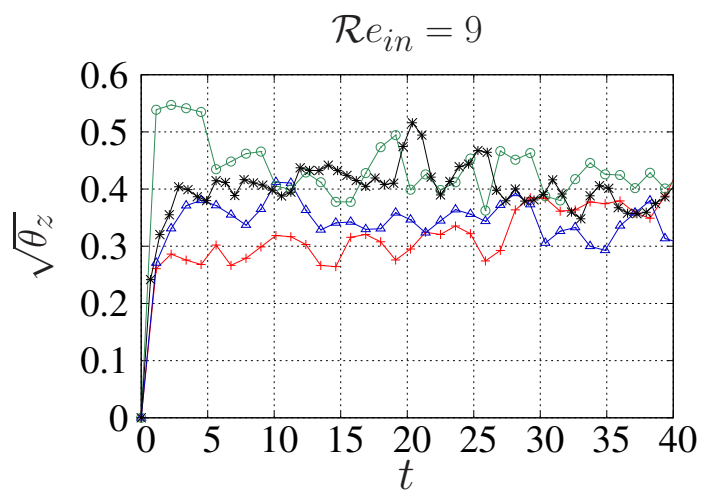

(c)

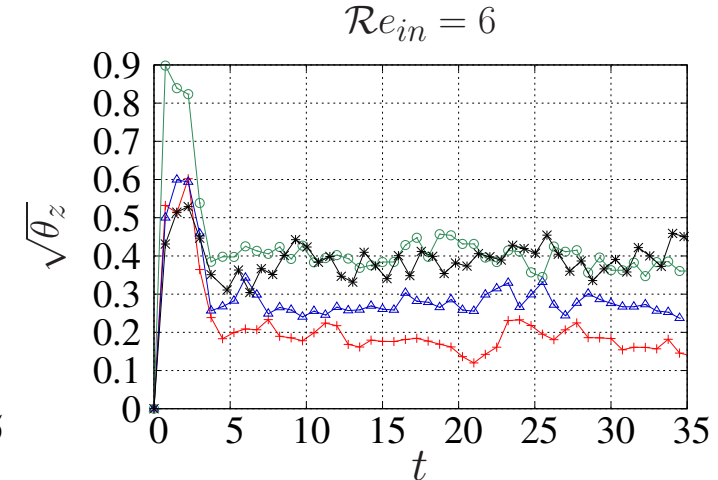

(b)

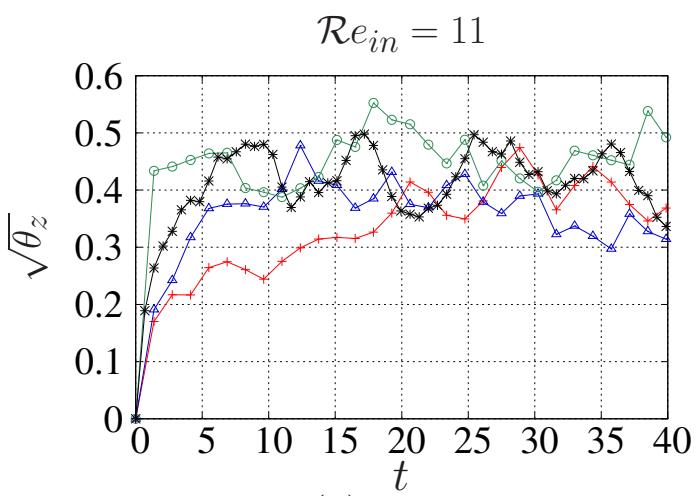

(d)

Figure 16. Evolution of axial granular temperature as a function of time in the liquid/solid regime with different inlet velocities. The definition of abbreviations is given in ТАВ. 2. Graph legends are the same in all figures and shown in figure (a) only. 
similar: both models predict an episodic slug formation at the vicinity of the entry which pushes the upper portion of the bed upward as in a piston-like fluidization. Particles above the slug smoothly rain down as the slug travels upward. Although the fluid field is clearly mollified in DEM-CFD simulations as compare to PRS, the meso-scale convective gradients are still well-predicted. We now focus on the influence of the proposed modifications for the meso-scale model in this high density ratio configuration. Starting from bed height evolution in FIG. 19(a), we do not observe any major difference in predictions by different DEM-CFD simulations. The mean bed height is mostly well-predicted by all variants of the meso-scale model, while employing a GK method captures marginally better large amplitude oscillations. DEM-CFD simulations

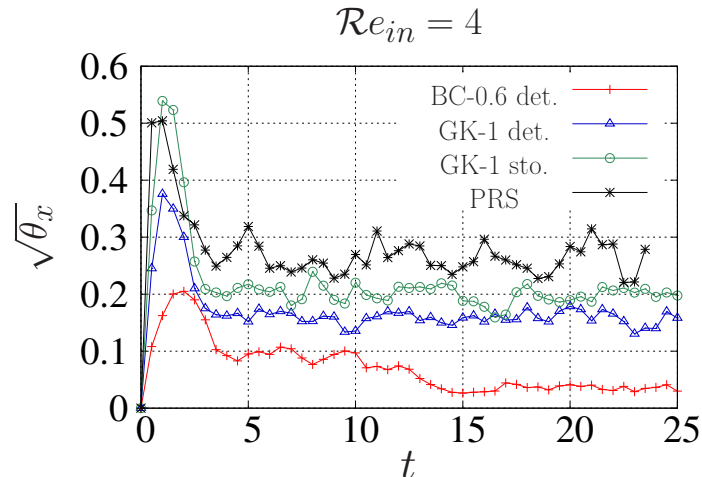

(a)

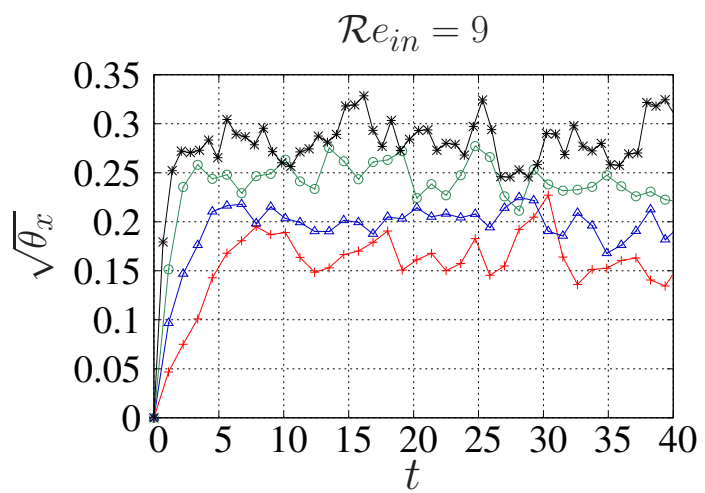

(c)

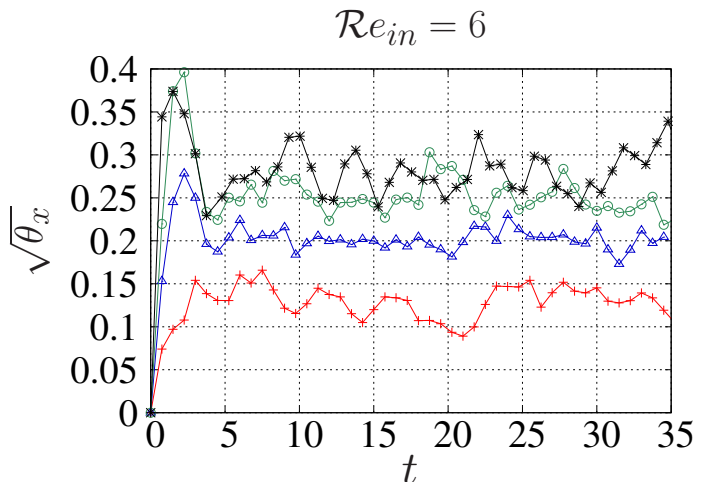

(b)

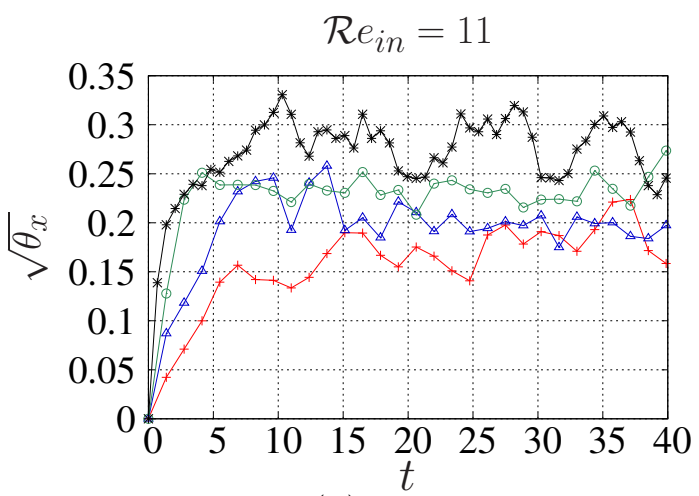

(d)

Figure 17. Evolution of transverse granular temperature as a function of time in the liquid/solid regime with different inlet velocities. The definition of abbreviations is given in ТАВ. 2. Graph legends are the same in all figures and shown in figure (a) only. 

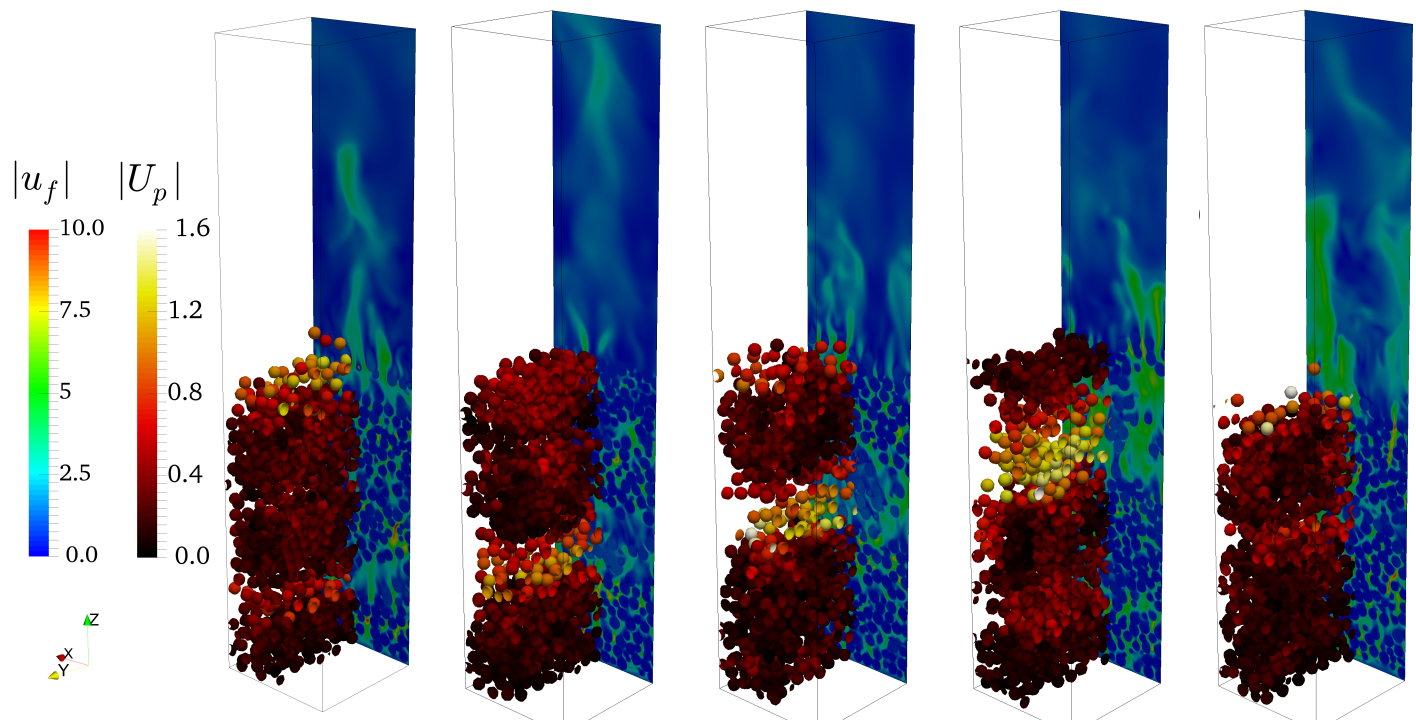

(a)
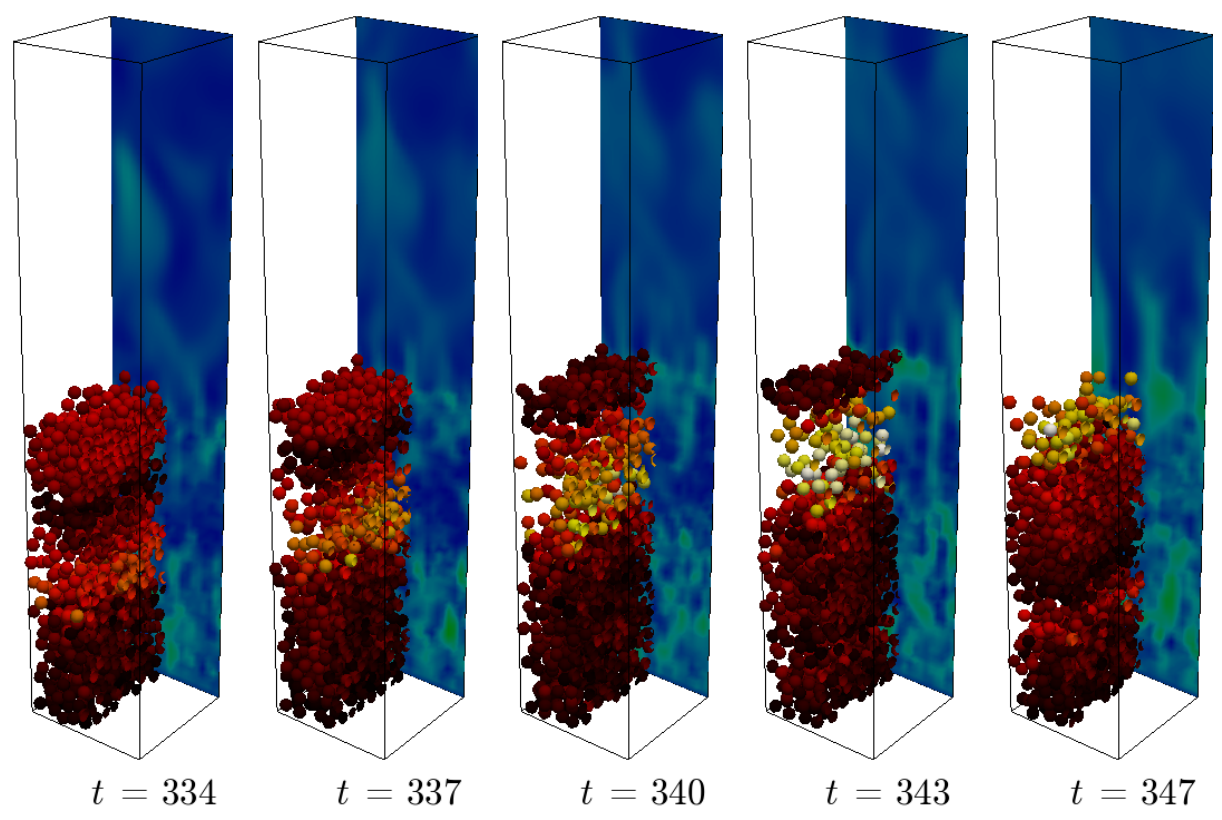

(b)

Figure 18. Bed evolution depicted by particles position and fluid velocity contours at a $y$-normal cut plane for (a) PRS and (b) GK-2 simulation with a deterministic drag, in the gas/solid regime. The definition of abbreviations is given in TAB. 2 .

predict the porosity profile within $5 \%$ error with respect to PRS, excluding the values near the bed upper limit and the entry. Deviations with respect to 
PRS in the range of $z \in[20: 28]$ is associated with the slug burst event near the upper surface of the bed and is mildly improved by employing the GK method with stochastic drag. In the vicinity of the entry, all variants of the meso-scale model underestimate porosity, as is also the case in the liquid/solid regime (FIG. 15).

As shown in FIG. 20, while all DEM-CFD variants are in good agreement with PRS results, the particles granular temperature is rather insensitive to DEMCFD modifications. FIGS. 20(a) and 20(b) show that employing a GK method improves DEM-CFD predictions of the mean axial granular temperature by about $10 \%$. In the transverse direction, employing the GK method and a finer fluid grid cell slightly overestimates the transverse granular temperature: $5 \%$ and $10 \%$ overestimation as compared to PRS, with a deterministic and stochastic drag, respectively.

\section{Discussion and perspectives}

In this study, we examined two directions of improvement of the meso-scale model. First, we employed a more sophisticated interphase coupling scheme

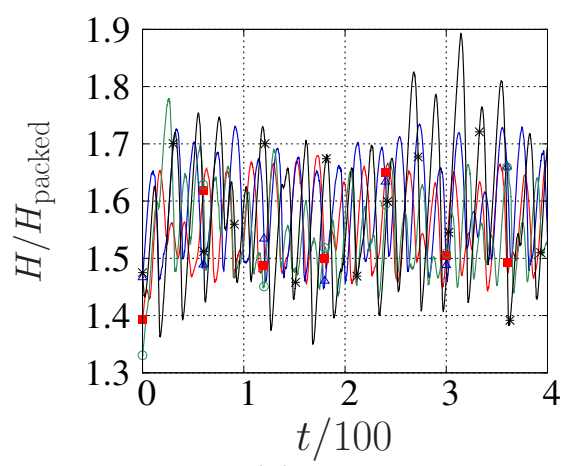

(a)

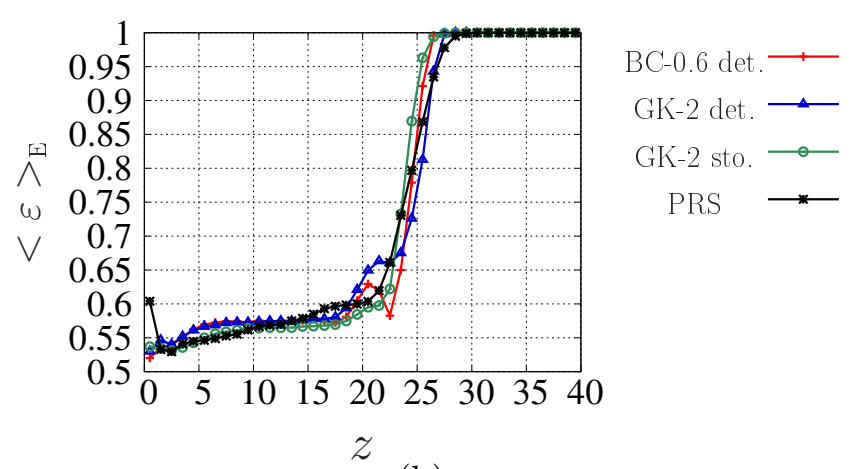

(b)

Figure 19. (a) Bed height expansion as a function of time and (b) fluid volume fraction axial profile, in the gas/solid regime. The definition of abbreviations is given in TAB. 2. 


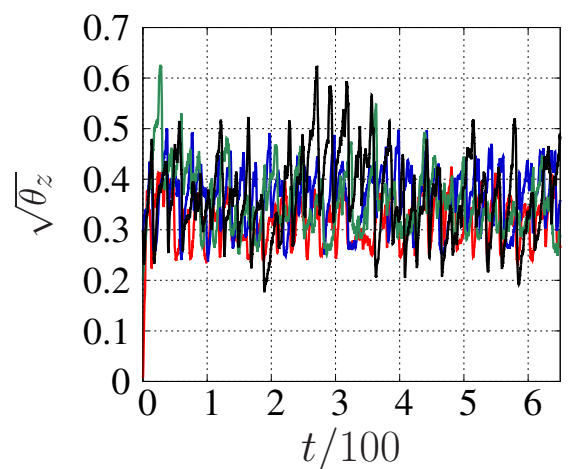

(a)

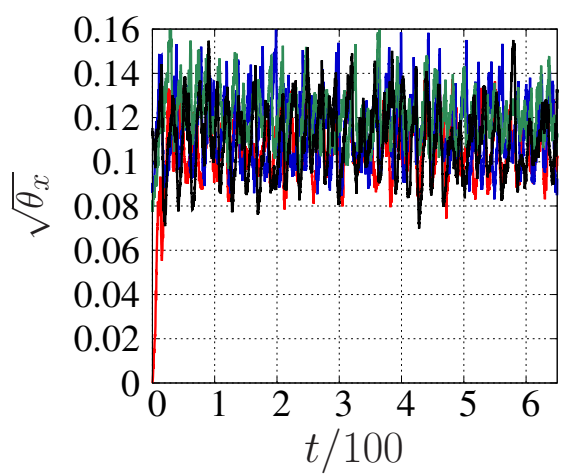

(c)

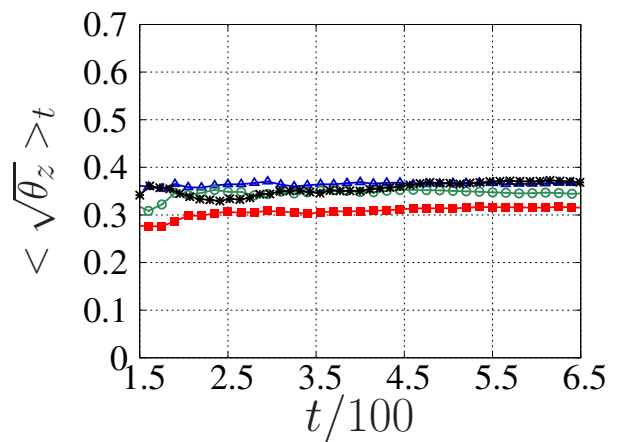

(b)

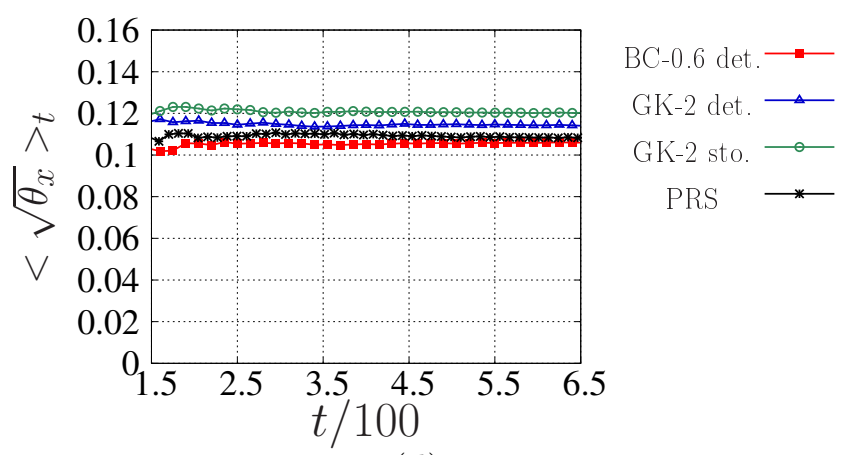

(d)

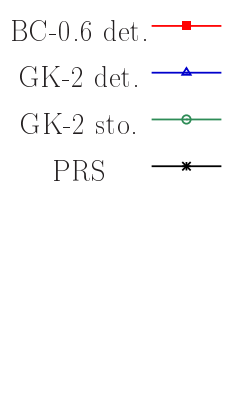

$$
\text { . }
$$

Figure 20. Time evolution of the instantaneous and time-averaged square root of the particles fluctuations in (a)-(b) the axial direction $z$ and (c)-(d) transverse direction $x$, in the gas/solid regime. The definition of abbreviations is given in TAB. 2 .

to decouple the averaging length scale from the fluid grid cell size. This socalled Gaussian kernel method, introduced by Capecelatro and Desjardins [2013], provides us with the flexibility of using a full range of grid sizes while guaranteeing a numerically stable solution. Second, we suggested an ad hoc remedy based on PRS results to capture the fluctuating nature of the drag force. We meticulously investigated the drag coefficient PDFs for given pairs of $\left(\mathcal{R} e_{p}, \varepsilon\right)$ and quantified their properties with respect to the locally filtered dimensionless variables. While there is no fundamental basis on the randomness of these sub-grid fluctuations, our numerical experiments based on PRS revealed that the shape of these PDFs is close to Gaussian particularly in the homogeneous liquid/solid regime. Thus, we proposed as a tentative solution a stochastic description for the drag force in the meso-scale model, assuming 
that sub-grid convective gradients can be simply represented by a localized random process. The characteristic time of the proposed stochastic drag is also extracted from PRS results. We adopted a first-order auto-regressive signal to introduce a fluctuating component in the drag force in the direction of the slip velocity. The magnitude of this fluctuating component is a function of the local $\mathcal{R} e_{p}$. We intentionally avoided to tackle the famous issue of the inconsistency between mean drag laws, as described by Kriebitzsch et al. [2013]: "the appropriateness of these static correlations for fluidized systems is a bit of a hit and miss affair". Among recent efforts in the literature to clarify these ambiguities, Rubinstein et al. [2016] highlighted the impact of Stokes number on drag force, while Tang et al. [2016] proposed a modified drag law taking into account particles mobility. In the present work, we turned our attention to a different question: assuming that we already have access to a mean drag correlation which is on average in accordance with PRS, can we capture the correct level of particles fluctuations with a meso-scale model? The present study has shown that the answer highly depends on the extent to which the already-resolved scales prevail.

We evaluated the contribution of the proposed improvements in two different regimes. We showed that in the homogeneously bubbling liquid/solid regime, both modifications have positively contributed to an enhanced DEM-CFD prediction of the particles granular temperature. By using a GK method and a stochastic drag, DEM-CFD simulations fully capture the axial particles granular temperature level as compared to PRS. In the transverse direction, we still observe minor discrepancies between DEM-CFD simulations and PRS. Our impression is that these minor differences stem from the direction of the fluctuating component. Recalling that the stochastic drag model mimics fluctuations originating from sub-grid scale convective gradients, it might not be 
necessarily collinear with the mean slip velocity. In a more realistic approach, one may consider a stochastic drag component which is not only fluctuating in magnitude, but also in direction.

In the gas/solid regime, we observed a highly heterogeneous fluidization with the dominance of meso-scale structures. For a given drag law (on average in agreement with PRS), the main characteristic phenomena of the system were well captured by a standard DEM-CFD model. Hence, we did not observe any favorable contribution of the stochastic drag to the DEM-CFD simulations in this system. In the presence of large and dominant meso-scale structures as, e.g., a slugging mechanism, the dynamics of particles is already well resolved by DEM-CFD simulations with GK (Gaussian kernel) and $\Delta x^{-1}$ in the range [1:3], and no stochastic drag, and is almost insensitive to local pseudo-random fluctuations. Please note that the grid resolution of our DEM-CFD simulations is by far more refined than what is used in coarse-grid simulations for industrial-scale applications. In DEM-CFD simulations of risers for instance, the fluid grid size is typically larger than the particles diameter by a factor of ten. In that situation, DEM-CFD simulations with grid resolutions equivalent to the ones we employed in the present study are considered as highly-resolved. A similar formalism is already adopted in the literature to improve the coarsegrid DEM-CFD simulations based on highly-resolved DEM-CFD simulations. In Andrews et al. [2005], the authors have already shown that although the main characteristics of gas/particle flows in a vertical riser are captured by the mean drag and a coarse-grid DEM-CFD simulation, a quantitative estimation requires a stochastic sub-grid correction.

In general, we believe that the present stochastic description of the drag force is particularly promising owing to its conceptual features: (i) all parameters 
can be extracted from PRS without any artificial fitting, and (ii) it takes into account the influence of already-resolved scales. Nonetheless, our approach is only an ad hoc remedy and is far from being a general solution to the classical problem of particle/fluid momentum transfer. As an engineering approach, one can perform various PRS with different dimensionless parameters in order to find the functional dependence of the stochastic model parameters on relevant dimensionless variables. In doing so, one may move to a tri-periodic box configuration in order to (i) decouple the dimensionless numbers of the system such as $\mathcal{R} e_{p}, \varepsilon$ and $\rho_{r}$, and (ii) eliminate the inlet boundary condition effects. Another parameter which needs to be taken into account in such an analysis is the size of the filter with respect to the particle size. The present study has shown that the filter size is an important parameter in the estimation of sub-grid scale fluctuations. This has been also reported by Capecelatro et al. [2015] in fully developed cluster-induced turbulence. Furthermore, we have developed and tested our stochastic drag model in a bi-periodic fluidized bed configuration only, i.e., a flow configuration without wall effects. Beyond exploring a wider range of dimensionless parameters and thereby examining other flow configurations as, e.g., dilute turbulent flows or clustering in moderately dilute flows, investigating the influence of wall boundary conditions is certainly another milestone to achieve a full description of the stochastic drag force.

Finally, another potential direction in improving drag laws (and in turn mesoscale models) is to better understand the physical origins of sub-grid fluctuations. We already know that an important source of drag modulation on a particle in a dense regime is the local fluctuations induced by neighboring particles. Thanks to the direct handling of particles motion in DEM-CFD models, detailed information about the local pore geometry is available. Hence, rather 
than merely computing the fluid volume fraction, more detailed information can be exploited by DEM-CFD models. For static particles, Akiki et al. [2017] has recently proposed a Faxén correction to quantify the fluid/particle force perturbation due to the presence of neighbors. The authors assumed that the pairwise interaction of particles can be linearly superposed even in fairly inertial regimes $\mathcal{R} e_{p}=170$. A major advantage of such approach is that due to its deterministic nature, it is applicable to a wide range of configurations.

\section{Appendices}

\section{A Description of drag correlations}

For the sake of consistency with the literature, all drag law formulations are given in dimensional variables and the Reynolds number is computed with the superficial slip velocity $\left|\boldsymbol{u}^{*}-\boldsymbol{U}^{*}\right| \varepsilon=V_{c}^{*}$ as a characteristic velocity and the particle diameter $d^{*}=L_{c}^{*}$ as a characteristic length:

$$
\mathcal{R} e_{p}=\frac{\rho_{f}^{*}\left|\boldsymbol{u}^{*}-\boldsymbol{U}^{*}\right| \varepsilon d^{*}}{\mu^{*}}
$$

Based on experiments for a wide range of Reynolds numbers and porosity, Di Felice [1994] proposed the following formulation:

$$
\begin{gathered}
\boldsymbol{F}_{d r a g}^{*}=0.5 C_{d} \rho_{f}^{*} \frac{\pi d^{* 2}}{4}\left|\boldsymbol{u}^{*}-\boldsymbol{U}^{*}\right|\left(\boldsymbol{u}^{*}-\boldsymbol{U}^{*}\right) \varepsilon^{-\chi} \\
\chi=3.7-0.65 \exp \left(-\frac{\left(1.5-\log _{10} \mathcal{R} e_{p}\right)^{2}}{2}\right) \\
C_{d}=\frac{24}{\mathcal{R} e_{p}}\left(1+0.15 \mathcal{R} e_{p}^{0.687}\right)
\end{gathered}
$$

where $C_{d}$ is the drag coefficient for a single unhindered particle for $\mathcal{R} e_{p}$ less than 1000 (Schiller and Naumann [1935]) and the pre-factor $\varepsilon^{-\chi}$ accounts for 
the presence of neighboring particles. This type of drag law was first proposed by Wen and Yu [1966], with a pre-factor $\varepsilon^{-3.7}$. However, the drag law was not applicable for $\varepsilon<0.8$. Thanks to the modifications of Di Felice [1994], the drag law is valid on a wide range of porosity.

The second drag law is based on the numerical simulations of Beetstra et al. [2007]:

$$
\begin{gathered}
\boldsymbol{F}_{\text {drag }}^{*}=3 \pi d^{*} \mu^{*}\left(\boldsymbol{u}^{*}-\boldsymbol{U}^{*}\right) g\left(\varepsilon, \mathcal{R} e_{p}\right) \\
g\left(\varepsilon, \mathcal{R} e_{p}\right)=\frac{10(1-\varepsilon)}{\varepsilon^{2}}+\varepsilon^{2}(1+1.5 \sqrt{1-\varepsilon}) \\
+\frac{0.413 \mathcal{R} e_{p}}{24 \varepsilon^{2}}\left(\frac{\varepsilon^{-1}+3 \varepsilon(1-\varepsilon)+8.4 \mathcal{R} e_{p}^{-0.343}}{1+10^{3(1-\varepsilon)} \mathcal{R} e_{p}^{-0.5-2(1-\varepsilon)}}\right)
\end{gathered}
$$

\section{B Mathematical description of the Gaussian Kernel interphase coupling method}

We first show how any Eulerian quantity and its derivatives can be reconstructed at a Lagrangian position. Starting with a Taylor expansion of an Eulerian quantity $\phi$ about the particle position:

$$
\phi(\boldsymbol{x})=\phi\left(\boldsymbol{r}_{i}\right)+\sum_{m}\left[\left(\boldsymbol{x}-\boldsymbol{r}_{i}\right) \frac{\partial \phi\left(\boldsymbol{r}_{i}\right)}{\partial x_{m}}\right]+\ldots
$$

We multiply both sides by a symmetric kernel function centered at the particle's position, $\mathcal{W}\left(\boldsymbol{x}-\boldsymbol{r}_{i}\right)$, and integrate over the flow domain $\Omega$ :

$$
\int_{\Omega} \phi(\boldsymbol{x}) \mathcal{W}\left(\boldsymbol{x}-\boldsymbol{r}_{i}\right) d \boldsymbol{x}=\phi\left(\boldsymbol{r}_{i}\right) \int_{\Omega} \mathcal{W}\left(\boldsymbol{x}-\boldsymbol{r}_{i}\right) d \boldsymbol{x}+\sum_{m}\left[\frac{\partial \phi\left(\boldsymbol{r}_{i}\right)}{\partial x_{m}} \int_{\Omega}\left(\boldsymbol{x}-\boldsymbol{r}_{i}\right) \mathcal{W}\left(\boldsymbol{x}-\boldsymbol{r}_{i}\right) d \boldsymbol{x}\right]+\ldots
$$


Neglecting all the derivative terms, we can have an estimation of $\phi\left(\boldsymbol{r}_{i}\right)$ :

$$
\phi\left(\boldsymbol{r}_{i}\right)=\frac{\int_{\Omega} \phi(\boldsymbol{x}) \mathcal{W}\left(\boldsymbol{x}-\boldsymbol{r}_{i}\right) d \boldsymbol{x}}{\int_{\Omega} \mathcal{W}\left(\boldsymbol{x}-\boldsymbol{r}_{i}\right) d \boldsymbol{x}}
$$

Due to the symmetry of the kernel, integral of the terms with odd-order derivatives are zero far from the boundaries. Hence the error produced by the truncation of the derivative terms is of order $\left|\boldsymbol{x}-\boldsymbol{r}_{i}\right|^{2}$ for the interior particles, and of order $\left|\boldsymbol{x}-\boldsymbol{r}_{i}\right|$ for particles near the boundaries. Now we estimate the first derivative of $\phi\left(\boldsymbol{x}_{i}\right)$. We replace $\mathcal{W}\left(\boldsymbol{x}-\boldsymbol{r}_{i}\right)$ with the derivative of the kernel $\frac{\partial \mathcal{W}\left(\boldsymbol{x}-\boldsymbol{r}_{i}\right)}{\partial \boldsymbol{x}_{m}}$ in equation B.2:

$$
\begin{aligned}
\int_{\Omega} \phi(\boldsymbol{x}) \frac{\partial \mathcal{W}\left(\boldsymbol{x}-\boldsymbol{r}_{i}\right)}{\partial \boldsymbol{x}_{m}} d \boldsymbol{x} & =\int_{\Omega} \phi\left(\boldsymbol{r}_{i}\right) \frac{\partial \mathcal{W}\left(\boldsymbol{x}-\boldsymbol{r}_{i}\right)}{\partial \boldsymbol{x}_{m}} d \boldsymbol{x}+ \\
\sum_{m} & {\left[\frac{\partial \phi\left(\boldsymbol{r}_{i}\right)}{\partial x_{m}} \int_{\Omega}\left(\boldsymbol{x}-\boldsymbol{r}_{i}\right) \frac{\partial \mathcal{W}\left(\boldsymbol{x}-\boldsymbol{r}_{i}\right)}{\partial \boldsymbol{x}_{m}} d \boldsymbol{x}\right]+\ldots }
\end{aligned}
$$

By neglecting second- and higher-order derivatives and rearranging equation B.5 we have:

$$
\int_{\Omega}\left[\phi(\boldsymbol{x})-\phi\left(\boldsymbol{r}_{i}\right)\right] \frac{\partial \mathcal{W}\left(\boldsymbol{x}-\boldsymbol{r}_{i}\right)}{\partial \boldsymbol{x}_{m}} d \boldsymbol{x}=\sum_{m}\left[\frac{\partial \phi\left(\boldsymbol{r}_{i}\right)}{\partial x_{m}} \int_{\Omega}\left(\boldsymbol{x}-\boldsymbol{r}_{i}\right) \frac{\partial \mathcal{W}\left(\boldsymbol{x}-\boldsymbol{r}_{i}\right)}{\partial \boldsymbol{x}_{m}} d \boldsymbol{x}\right]
$$

In a 1-D system, B.6 directly yields to an expression for the derivative:

$$
\frac{d \phi\left(\boldsymbol{r}_{i}\right)}{d x_{m}}=\frac{\int_{\Omega}\left[\phi(\boldsymbol{x})-\phi\left(\boldsymbol{r}_{i}\right)\right] \frac{d \mathcal{W}\left(\boldsymbol{x}-\boldsymbol{r}_{i}\right)}{d \boldsymbol{x}_{m}} d \boldsymbol{x}}{\int_{\Omega}\left(\boldsymbol{x}-\boldsymbol{r}_{i}\right) \frac{d \mathcal{W}\left(\boldsymbol{x}-\boldsymbol{r}_{i}\right)}{d \boldsymbol{x}_{m}} d \boldsymbol{x}}, \quad m=1
$$

In order to avoid singular values in the denominator, the $\frac{d \mathcal{W}\left(\boldsymbol{x}-\boldsymbol{r}_{i}\right)}{d \boldsymbol{x}_{m}}$ needs to be an anti-symmetric function. Similar to 17 , the truncation error is of order $\left|\boldsymbol{x}-\boldsymbol{r}_{i}\right|^{2}$ for the interior particles, and of order $\left|\boldsymbol{x}-\boldsymbol{r}_{i}\right|$ for particles near 
the boundaries. In a 3-D system, B.6 represents a set of linear equations for different components of the derivative:

$$
\boldsymbol{A} \boldsymbol{X}=\boldsymbol{B}
$$

$$
\boldsymbol{A}=\left[\begin{array}{l}
\int_{\Omega}\left(x_{1}-r_{i, 1}\right) \frac{\partial \mathcal{W}\left(\boldsymbol{x}-\boldsymbol{r}_{i}\right)}{\partial x_{1}} d \boldsymbol{x} \int_{\Omega}\left(x_{2}-r_{i, 2}\right) \frac{\partial \mathcal{W}\left(\boldsymbol{x}-\boldsymbol{r}_{i}\right)}{\partial x_{1}} d \boldsymbol{x} \int_{\Omega}\left(x_{3}-r_{i, 3}\right) \frac{\partial \mathcal{W}\left(\boldsymbol{x}-\boldsymbol{r}_{i}\right)}{\partial x_{1}} d \boldsymbol{x} \\
\int_{\Omega}\left(x_{1}-r_{i, 1}\right) \frac{\partial \mathcal{W}\left(\boldsymbol{x}-\boldsymbol{r}_{i}\right)}{\partial x_{2}} d \boldsymbol{x} \int_{\Omega}\left(x_{2}-r_{i, 2}\right) \frac{\partial \mathcal{W}\left(\boldsymbol{x}-\boldsymbol{r}_{i}\right)}{\partial x_{2}} d \boldsymbol{x} \int_{\Omega}\left(x_{3}-r_{i, 3}\right) \frac{\partial \mathcal{W}\left(\boldsymbol{x}-\boldsymbol{r}_{i}\right)}{\partial x_{2}} d \boldsymbol{x} \\
\int_{\Omega}\left(x_{1}-r_{i, 1}\right) \frac{\partial \mathcal{W}\left(\boldsymbol{x}-\boldsymbol{r}_{i}\right)}{\partial x_{3}} d \boldsymbol{x} \int_{\Omega}\left(x_{2}-r_{i, 2}\right) \frac{\partial \mathcal{W}\left(\boldsymbol{x}-\boldsymbol{r}_{i}\right)}{\partial x_{3}} d \boldsymbol{x} \int_{\Omega}\left(x_{3}-r_{i, 3}\right) \frac{\partial \mathcal{W}\left(\boldsymbol{x}-\boldsymbol{r}_{i}\right)}{\partial x_{3}} d \boldsymbol{x}
\end{array}\right]
$$

$$
\boldsymbol{X}=\left[\begin{array}{c}
\frac{\partial \phi\left(\boldsymbol{r}_{i}\right)}{\partial x_{1}} \\
\frac{\partial \phi\left(\boldsymbol{r}_{i}\right)}{\partial x_{2}} \\
\frac{\partial \phi\left(\boldsymbol{r}_{i}\right)}{\partial x_{3}}
\end{array}\right], \quad \boldsymbol{B}=\left[\begin{array}{l}
\int_{\Omega}\left[\phi(\boldsymbol{x})-\phi\left(\boldsymbol{r}_{i}\right)\right] \frac{\partial \mathcal{W}\left(\boldsymbol{x}-\boldsymbol{r}_{i}\right)}{\partial \boldsymbol{x}_{1}} d \boldsymbol{x} \\
\int_{\Omega}\left[\phi(\boldsymbol{x})-\phi\left(\boldsymbol{r}_{i}\right)\right] \frac{\partial \mathcal{W}\left(\boldsymbol{x}-\boldsymbol{r}_{i}\right)}{\partial \boldsymbol{x}_{2}} d \boldsymbol{x} \\
\int_{\Omega}\left[\phi(\boldsymbol{x})-\phi\left(\boldsymbol{r}_{i}\right)\right] \frac{\partial \mathcal{W}\left(\boldsymbol{x}-\boldsymbol{r}_{i}\right)}{\partial \boldsymbol{x}_{3}} d \boldsymbol{x}
\end{array}\right]
$$

In practice, the matrix inversion is done only once and the solution is explicitly derived. Similarly, higher-order derivatives can be achieved by replacing the kernel with its high-order derivatives in equation B.2 and following the same procedure. In our formulations, however, we do not need such expressions.

So far, we have shown how to reconstruct a particle property from the background fluid field. For the reverse operation, i.e., projecting the particles information on the Eulerian field, the procedure is essentially similar. We start with a Taylor expansion of a particle quantity $\phi$ about an arbitrary Eulerian position $\phi\left(\boldsymbol{x}_{j}\right)$ :

$$
\phi(\boldsymbol{x})=\phi\left(\boldsymbol{x}_{j}\right)+\sum_{m}\left[\left(\boldsymbol{x}-\boldsymbol{x}_{j}\right) \frac{\partial \phi\left(\boldsymbol{x}_{i}\right)}{\partial x_{m}}\right]+\ldots
$$


We multiply both sides by a symmetric kernel function centered at the position of the selected Eulerian point, $\mathcal{W}\left(\boldsymbol{x}-\boldsymbol{x}_{j}\right)$, and integrate over $\Omega . V_{p}^{\text {total }}=$ $\bigcup_{i=1}^{N p} V_{i}$. By neglecting all the derivative terms we have:

$$
\phi\left(\boldsymbol{x}_{j}\right)=\frac{\int_{\Omega} \phi(\boldsymbol{x}) \mathcal{W}\left(\boldsymbol{x}-\boldsymbol{x}_{j}\right) d \boldsymbol{x}}{\int_{\Omega} \mathcal{W}\left(\boldsymbol{x}-\boldsymbol{x}_{j}\right) d \boldsymbol{x}}
$$

Given that $\Omega=\Omega_{f} \cup V_{p}^{\text {total }}$ where $V_{p}^{\text {total }}=\bigcup_{i=1}^{N p} V_{i}$, B.12 expands as:

$$
\phi\left(\boldsymbol{x}_{j}\right)=\frac{\int_{\Omega_{f}} \phi(\boldsymbol{x}) \mathcal{W}\left(\boldsymbol{x}-\boldsymbol{x}_{j}\right) d \boldsymbol{x}+\int_{V_{p}^{\text {total }}} \phi(\boldsymbol{x}) \mathcal{W}\left(\boldsymbol{x}-\boldsymbol{x}_{j}\right) d \boldsymbol{x}}{\int_{\Omega} \mathcal{W}\left(\boldsymbol{x}-\boldsymbol{x}_{j}\right) d \boldsymbol{x}}
$$

Given that any particle variable is constant over the respective particle domain and is zero over the fluid domain, B.13 can be transformed to:

$$
\phi\left(\boldsymbol{x}_{j}\right)=\frac{\sum_{i=1}^{N_{p}} \phi\left(\boldsymbol{r}_{i}\right) \mathcal{W}\left(\boldsymbol{r}_{i}-\boldsymbol{x}_{j}\right) V_{p, i}}{\int_{\Omega} \mathcal{W}\left(\boldsymbol{x}-\boldsymbol{x}_{j}\right) d \boldsymbol{x}}
$$

Although not required in our formulations, all the derivatives of a particle property at an Eulerian position can also be achieved in a similar fashion.

\section{Grid and kernel size refinement analysis in DEM-CFD simula- tions}

We choose a range of $0.5 \leq \Delta x^{-1} \leq 3$ to investigate the impact of grid resolution on computed solutions. We denote $\delta_{\text {sup }}=2 \delta_{0.5}$ as the kernel support size. In our grid-refinement analysis, we set $\delta_{\text {sup }}=2$, while the influence of $\delta_{\text {sup }}$ 
on the predicted solution is subsequently investigated. For the sake of brevity, we limit our analysis only to one set of parameters: $\mathcal{R} e_{i n}=6, \rho_{r}=10$.

We study the sensitivity of particle fluctuations to grid resolution in DEMCFD simulations in Fig. B.1. As a first remark, the successive grid refinement enhances the level of particles fluctuations in both axial and transverse directions up to one grid cell per diameter. We see a non-negligible improvement of the predicted solution by using a GK method and a more refined grid size. These improvements are particularly noticeable in the transverse direction, where particles motion is mostly driven by fluid fluctuations rather than mean flow. Solutions predicted by GK-1 to GK-3 are almost insensitive to grid

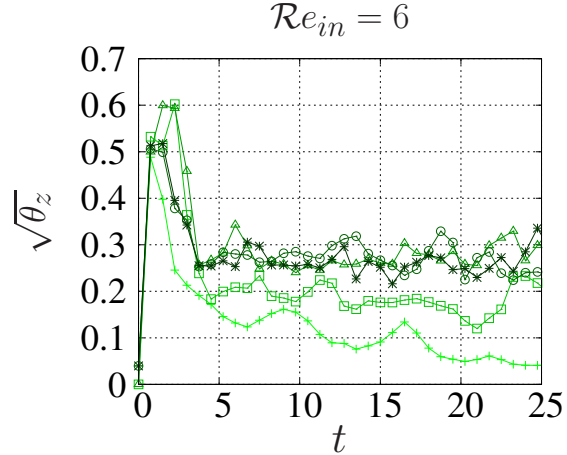

(a)

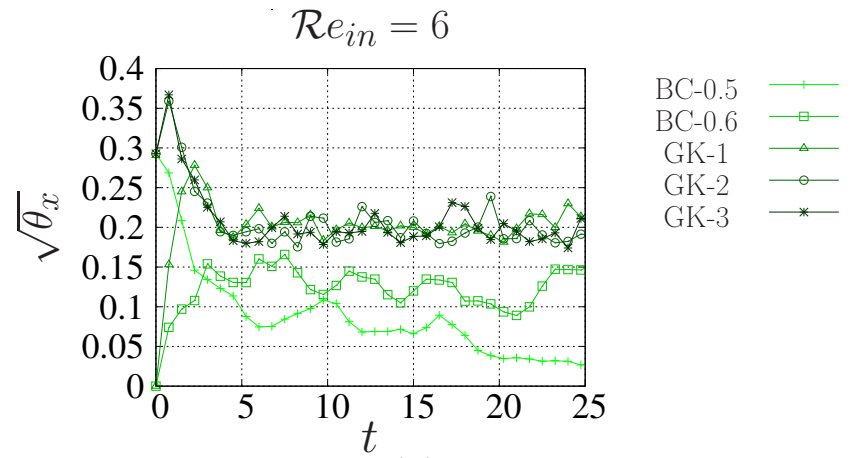

(b)

Figure B.1. Grid refinement analysis for DEM-CFD simulations. Evolution of (a) axial and (b) transverse granular temperature as a function of time in the liquid/solid regime.

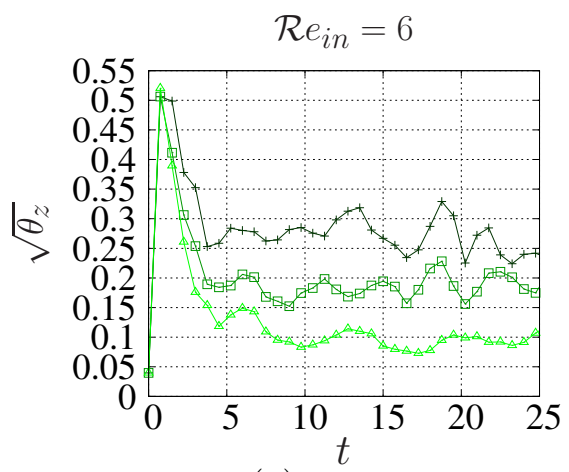

(a)

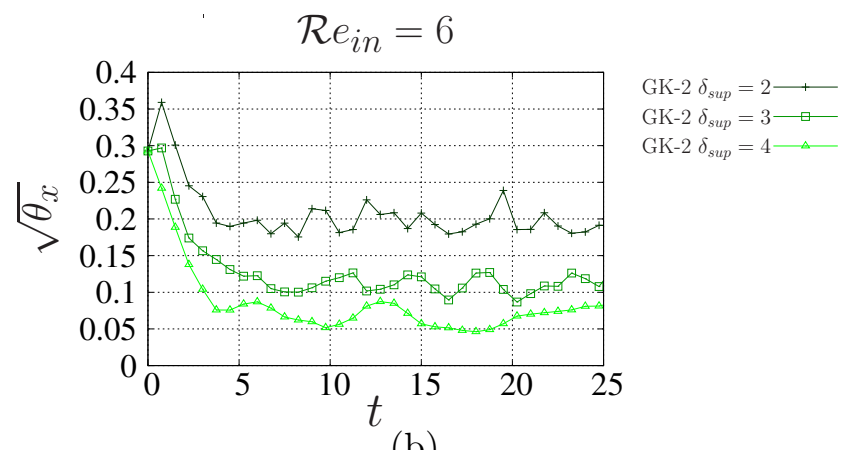

(b)

Figure B.2. Kernel support size refinement analysis for DEM-CFD simulations. Evolution of (a) axial and (b) transverse granular temperature as a function of time in the liquid/solid regime. 
resolution. This was predictable since DEM-CFD simulations are intrinsically controlled by the averaging operation. When the BC method is adopted, the grid refinement implicitly influences the local averaging length scale and in turn the level of fluctuations. With the GK method, however, the local averaging is decoupled from the grid size thanks to a smooth filtering kernel and its length scale is hence adjusted by the kernel support.

Finally, we study the impact of the kernel support size $\delta_{\text {sup }}$ on the predicted solution. We choose the case with 2 grid points per diameter, GK-2, and perform different simulations for a range of kernel support sizes. A kernel support size of less than two diameters is not acceptable since it violates the basic principle of locally averaged Navier-Stokes equations on which our model is based: the local averaging volume should be about an order of magnitude larger than the particle diameter. The upper bound is also limited by the transverse domain size which is 8 diameters. We hence choose $2 \leq \delta_{\text {sup }} \leq 4$ as a reasonable range for the kernel support size analysis. FIG. B.2 show how kernel size influences particles granular temperature in both axial and transverse directions. With an increasing kernel size, we further suppress local fluid fluctuations and in turn drag force agitations. As a result, particles granular temperature diminishes as kernel size increases. As a general trend, employing a large kernel support with the GK method seems to provide similar results to those provided by the $\mathrm{BC}$ method and large grid sizes. In other words, refining the grid size and filtering it at the projection step is redundant from a modeling standpoint. We hence choose a kernel support with $\delta_{\text {sup }}=2$ for the rest of our analysis. 


\section{References}

G. Akiki, T. L. Jackson, and S. Balachandar. Pairwise interaction extended point-particle model for a random array of monodisperse spheres. Journal of Fluid Mechanics, 813:882-928, 2017.

T. B. Anderson and R. Jackson. Fluid mechanical description of fluidized beds. Equations of motion. Industrial \& Engineering Chemistry Fundamentals, 6 (4):527-539, 1967.

I.V. Andrews, T. Arthur, P. N. Loezos, and S. Sundaresan. Coarse-grid simulation of gas-particle flows in vertical risers. Industrial $\&$ engineering chemistry research, 44(16):6022-6037, 2005.

R. Beetstra, M.A. Van der Hoef, and J.A.M. Kuipers. Drag force of intermediate reynolds number flow past mono-and bidisperse arrays of spheres. AIChE Journal, 53(2):489-501, 2007.

S. Benyahia, M. Syamlal, and T. J. O'Brien. Evaluation of boundary conditions used to model dilute, turbulent gas/solids flows in a pipe. Powder Technology, 156(2):62-72, 2005.

M. Bernard, E. Climent, and A. Wachs. Controlling the quality of two-way Euler/Lagrange numerical modeling of bubbling and spouted fluidized beds dynamics. Industrial \& Engineering Chemistry Research, 56(1):368-386, 2016 .

A. S. Berrouk, D. Laurence, J. J. Riley, and D. E. Stock. Stochastic modelling of inertial particle dispersion by subgrid motion for LES of high Reynolds number pipe flow. Journal of Turbulence, 8:N50, 2007.

S. Bogner, S. Mohanty, and U. Rüde. Drag correlation for dilute and moderately dense fluid-particle systems using the lattice Boltzmann method. International Journal of Multiphase Flow, 68:71-79, 2015.

G.E.P. Box, G. M. Jenkins, G. C. Reinsel, and G. M. Ljung. Time series 
analysis: forecasting and control. John Wiley \& Sons, 2015.

J. Capecelatro and O. Desjardins. An Euler-Lagrange strategy for simulating particle-laden flows. Journal of Computational Physics, 238:1-31, 2013.

J. Capecelatro, O. Desjardins, and R. O. Fox. On fluid-particle dynamics in fully developed cluster-induced turbulence. Journal of Fluid Mechanics, 780:578-635, 2015.

C. T. Crowe, M. Sommerfeld, and Y. Tsuji. Multiphase flows with droplets and particles. CRC press, 1997.

R. Di Felice. The voidage function for fluid-particle interaction systems. International Journal of Multiphase Flow, 20(1):153-159, 1994.

T. D. Dreeben and S. B. Pope. Probability density function/Monte Carlo simulation of near-wall turbulent flows. Journal of Fluid Mechanics, 357: 141-166, 1998.

S. Ergun. Fluid flow through packed columns. Chemical engineering progress, 48, 1952 .

A. Esteghamatian, M. Bernard, M. Lance, A. Hammouti, and A. Wachs. Micro/meso simulation of a fluidized bed in a homogeneous bubbling regime. International Journal of Multiphase Flow, 92:93-111, 2017a.

A. Esteghamatian, A. Hammouti, M. Lance, and A. Wachs. Particle resolved simulations of liquid/solid and gas/solid fluidized beds. Physics of Fluids, 29(3):033302, 2017b.

L-S Fan and C. Zhu. Principles of gas-solid flows. Cambridge University Press, 2005.

P. Fede, O. Simonin, P. Villedieu, and K. D. Squires. Stochastic modeling of the turbulent subgrid fluid velocity along inertial particle trajectories. Proceeding of the Summer Program Center for Turbulence Research Stanford University, Stanford, CA, July, pages 247-258, 2006.

R.O. Fox. Large-eddy-simulation tools for multiphase flows. Annual Review 
of Fluid Mechanics, 44:47-76, 2012.

R. Gatignol. The faxén formulas for a rigid particle in an unsteady nonuniform stokes-flow. Journal de Mécanique Théorique et Appliquée, 2(2): 143-160, 1983.

D. Gidaspow. Multiphase flow and fluidization: continuum and kinetic theory descriptions. Academic press, 1994.

R. Glowinski, T. W. Pan, T. I. Hesla, D. D. Joseph, and J. Periaux. A fictitious domain approach to the direct numerical simulation of incompressible viscous flow past moving rigid bodies: application to particulate flow. Journal of Computational Physics, 169(2):363-426, 2001.

R. J. Hill, D. L. Koch, and A. J. C. Ladd. Moderate-reynolds-number flows in ordered and random arrays of spheres. Journal of Fluid Mechanics, 448: 243-278, 2001.

L. Huilin and D. Gidaspow. Hydrodynamics of binary fluidization in a riser: CFD simulation using two granular temperatures. Chemical Engineering Science, 58(16):3777-3792, 2003.

J. T. Jenkins and M. Y. Louge. On the flux of fluctuation energy in a collisional grain flow at a flat, frictional wall. Physics of Fluids (1994-present), 9(10): 2835-2840, 1997.

S. H. L. Kriebitzsch, M. A. Van der Hoef, and J. A. M. Kuipers. Fully resolved simulation of a gas-fluidized bed: a critical test of dem models. Chemical Engineering Science, 91:1-4, 2013.

T. Li, J. Grace, and X. Bi. Study of wall boundary condition in numerical simulations of bubbling fluidized beds. Powder Technology, 203(3):447-457, 2010.

J.M. Link, L.A. Cuypers, N.G. Deen, and J.A.M. Kuipers. Flow regimes in a spout-fluid bed: A combined experimental and simulation study. Chemical Engineering Science, 60(13):3425-3442, 2005. 
M. R. Maxey and J. J. Riley. Equation of motion for a small rigid sphere in a nonuniform flow. Physics of Fluids (1958-1988), 26(4):883-889, 1983.

M. Muradoglu, P. Jenny, S. B. Pope, and D. A. Caughey. A consistent hybrid finite-volume/particle method for the PDF equations of turbulent reactive flows. Journal of Computational Physics, 154(2):342-371, 1999.

B. Oesterle and A. Petitjean. Simulation of particle-to-particle interactions in gas solid flows. International journal of multiphase flow, 19(1):199-211, 1993.

P. Pepiot and O. Desjardins. Numerical analysis of the dynamics of two-and three-dimensional fluidized bed reactors using an Euler-Lagrange approach. Powder Technology, 220:104-121, 2012.

S. B. Pope. Lagrangian PDF methods for turbulent flows. Annual review of fluid mechanics, 26(1):23-63, 1994.

A. Rakotonirina and A. Wachs. Grains3D, a flexible DEM approach for particles of arbitrary convexshape - Part II: parallel implementation and scalable performance. in revision in Powder Technology, 2017.

A. Rakotonirina, J.-Y. Delenne, F. Radjai, and A. Wachs. Grains3D, a flexible DEM approach for particles of arbitrary convexshape - Part III: extension to non convex particles. submitted to Computational Particle Mechanics, 2017.

G. J. Rubinstein, J. J. Derksen, and S. Sundaresan. Lattice Boltzmann simulations of low-Reynolds-number flow past fluidized spheres: effect of Stokes number on drag force. Journal of Fluid Mechanics, 788:576-601, 2016.

L. Schiller and Z. Naumann. A drag coefficient correlation. Vdi Zeitung, 77 $(318): 51,1935$.

M. Sommerfeld and G. Zivkovic. Recent advances in the numerical simulation of pneumatic conveying through pipe systems. Computational methods in applied sciences, 201, 1992. 
S. Subramaniam. Statistical representation of a spray as a point process. Physics of Fluids (1994-present), 12(10):2413-2431, 2000.

S. Subramaniam, M. Mehrabadi, J. Horwitz, and A. Mani. Developing improved Lagrangian point particle models of gas-solid flow from particleresolved direct numerical simulation. Studying Turbulence Using Numerical Simulation Databases-XV, Proceedings of the CTR, pages 5-14, 2014.

Y. Tang, E.A.J.F. Peters, J.A.M. Kuipers, S.H.L. Kriebitzsch, and M.A. Hoef. A new drag correlation from fully resolved simulations of flow past monodisperse static arrays of spheres. AIChE journal, 61(2):688-698, 2015.

Y. Tang, Y. M. Lau, N. G. Deen, E. A. J. F. Peters, and J. A. M. Kuipers. Direct numerical simulations and experiments of a pseudo-2D gas-fluidized bed. Chemical Engineering Science, 143:166-180, 2016.

S. Tenneti and S. Subramaniam. Particle-resolved direct numerical simulation for gas-solid flow model development. Annual review of fluid mechanics, 46 : 199-230, 2014.

S. Tenneti, R. Garg, and S. Subramaniam. Drag law for monodisperse gassolid systems using particle-resolved direct numerical simulation of flow past fixed assemblies of spheres. International Journal of Multiphase flow, 37(9): 1072-1092, 2011.

S. Tenneti, M. Mehrabadi, and S. Subramaniam. Stochastic Lagrangian model for hydrodynamic acceleration of inertial particles in gas-solid suspensions. Journal of Fluid Mechanics, 788:695-729, 2016.

Y. Tsuji, T. Kawaguchi, and T. Tanaka. Discrete particle simulation of twodimensional fluidized bed. Powder technology, 77(1):79-87, 1993.

M. A. Van der Hoef, M. van Sint Annaland, N. G. Deen, and J. A. M. Kuipers. Numerical Simulation of Dense Gas-Solid Fluidized Beds: A Multiscale Modeling Strategy. Annual Review of Fluid Mechanics, 70:40-47, 2008. 
A. Wachs, L. Girolami, G. Vinay, and G. Ferrer. Grains3D, a flexible DEM approach for particles of arbitrary convexshape - Part I: Numerical model and validations. Powder Technology, 224:374-389, 2012.

A. Wachs, A. Hammouti, G. Vinay, and M. Rahmani. Accuracy of finite volume/staggered grid distributed lagrange multiplier/fictitious domain simulations of particulate flows. Computers \& Fluids, 115:154-172, 2015.

C. Wen and Y.H. Yu. Mechanics of fluidization. In Chem. Eng. Prog. Symp. Ser., volume 62, page 100, 1966. 


\section{List of Tables}

$1 \quad$ Physical parameters of the system 18

2 Numerical parameters of DEM-CFD and PR simulations. 


\section{List of Figures}

1 Bounding Cube averaging method in meso-scale model:

(a) Lagrange $\rightarrow$ Euler and (b) Euler $\rightarrow$ Lagrange information transfer.

2 Gaussian Kernel averaging method: Illustration of (a) distribution of a particle property over the neighboring cells and (b) performance of the projection kernel close to a boundary. In (b), the real and image particles are in solid green, and red hashed pattern, respectively. The solid lines represent the projection kernel of the real and image particles, while the red dashed line is the superposition of both kernels.

3 A Gaussian filtering kernel and its derivative. $\delta_{0.5}$ denotes the width of the kernel where it reaches $50 \%$ of its maximum value.

4 Flow domain and layout of particles at $t=0$ in (a) liquid/solid configurations and (b) gas/solid configurations. 
5 (a) Reconstruction of the PRS fluid field (black cells)

at the DEM-CFD grid cell centers (white cells/points).

Volume-averaged values are then linearly interpolated at the particle center. (b) Decomposition of the hydrodynamic force acting on a particle. The plane $P$ is perpendicular to the slip velocity vector $\left(\boldsymbol{U}_{\text {slip }}=\boldsymbol{u}-\boldsymbol{U}\right) . \boldsymbol{F}_{f p}$ denotes the total hydrodynamic force, $\boldsymbol{F}_{\text {drag }}$ and $\boldsymbol{F}_{\text {drift }}$ are the components in parallel and perpendicular directions with respect to the slip velocity vector.

6 Probability Density Function (PDF) of drag coefficient, $C_{d}$, for a given pair of $\mathcal{R} e_{p}$ and $\varepsilon$ computed from the PRS results and different drag laws (Beetstra et al. [2007], Huilin and Gidaspow [2003], Di Felice [1994]) in (a) liquid/solid regimes and (b) gas/solid regimes. PRS results are shown as a PDF, and the deterministic drag correlations are shown by a Dirac function. The uncertainty of the values computed by the drag correlations are shown by solid lines and corresponds to the relative uncertainty tolerance of $1 \%$ in $\mathcal{R} e_{p}$ and $\varepsilon$.

7 Variation of the drag coefficient PDF for a given pair of $\mathcal{R} e_{p}$ and $\varepsilon$ with respect to the number of realizations in (a) liquid/solid regimes and (c) gas/solid regimes. (b) and (d) show the dependence of the mean and standard deviation values to the number of realizations, in liquid/solid regimes and gas/solid regimes respectively. 
8 Drag coefficient as a function of $\mathcal{R} e_{p}$ and for different $\varepsilon$. Drag correlations $C_{d}^{\text {correl }}$ in the literature (Beetstra et al. [2007] and Di Felice [1994] for (a) liquid/solid regimes and (b) gas/solid regimes, respectively) and drag coefficients $C_{d}^{\mathrm{PRS}}$ predicted by PRS are represented by lines and symbols, respectively.

9 Standard deviation of the drag coefficient in liquid/solid regimes as a function of $\mathcal{R} e_{p}$ and for different $\varepsilon$ : (a) absolute value and (b) value normalized by mean drag coefficient.

10 Standard deviation of the drag coefficient in the gas/solid regime as a function of $\mathcal{R} e_{p}$ and for different $\varepsilon$ : (a) absolute value and (b) value normalized by mean drag coefficient.

11 PDF of the fluid/particle force normalized by the buoyancy force over (a) ensemble of particles (spatial) or (b) particle's trajectory (temporal) in liquid/solid regimes.

12 Auto-correlation function of the drag force magnitude as a function of the dimensionless separation time in (a) liquid/solid regimes and (b) gas/solid regimes.

13 Correlation time $\mathcal{T}$ as a function of inlet Reynolds number in liquid/solid regimes. 
14 (a) An example of $C_{d}^{\prime}$ signal as a function of time and (b) autocorrelation function of a stochastic drag coefficient $C_{d}^{s t o}$ and its fluctuating component, $C_{d}^{\prime}$ (c.f. (33)) compared to a classical drag coefficient without stochastic component, $C_{d}^{\text {det }}$. The $x$-axis is normalized by the integral time, $\mathcal{T}$ (defined in (30) and (31)), corresponding to the respective curve. Both plots correspond to a DEM-CFD simulation in a liquid/solid regime.

15 Fluid volume fraction axial profile in the liquid/solid regime with different inlet velocities. The definition of abbreviations is given in TAB. 2 .

16 Evolution of axial granular temperature as a function of time in the liquid/solid regime with different inlet velocities. The definition of abbreviations is given in TAB. 2. Graph legends are the same in all figures and shown in figure (a) only.

17 Evolution of transverse granular temperature as a function of time in the liquid/solid regime with different inlet velocities. The definition of abbreviations is given in TAB. 2. Graph legends are the same in all figures and shown in figure (a) only.

18 Bed evolution depicted by particles position and fluid velocity contours at a $y$-normal cut plane for (a) PRS and (b) GK-2 simulation with a deterministic drag, in the gas/solid regime. The definition of abbreviations is given in TAB. 2 . 
19 (a) Bed height expansion as a function of time and (b) fluid volume fraction axial profile, in the gas/solid regime. The definition of abbreviations is given in TAB. 2 .

20 Time evolution of the instantaneous and time-averaged square root of the particles fluctuations in (a)-(b) the axial direction $z$ and $(\mathrm{c})-(\mathrm{d})$ transverse direction $x$, in the gas/solid regime. The definition of abbreviations is given in TAB. 2 .

B.1 Grid refinement analysis for DEM-CFD simulations. Evolution of (a) axial and (b) transverse granular temperature as a function of time in the liquid/solid regime.

B.2 Kernel support size refinement analysis for DEM-CFD simulations. Evolution of (a) axial and (b) transverse granular temperature as a function of time in the liquid/solid regime. 